\title{
Cohomology and Extensions of von Neumann Algebras. II
}

By

\author{
Colin. E. SUTHERLAND*1)
}

\begin{abstract}
We develop a theory of extensions of von Neumann algebras by locally compact groups of automorphisms. The emphasis is on the description (from an algebraic point of view) of those extensions of a given von Neumann algebra by a given group which determine a fixed homomorphism from the group into the outer automorphism classes of the given algebra. Thus the study of such homomorphisms occupies a substantial part of the paper; for a large class of examples we are able to determine when such a homomorphism is split, and give a simple algebraic description of the extensions. We then give necessary and sufficient conditions (of an analytic nature) for an extension to be equivalent to a twisted crossed product extension, and give some applications to the study of representations of certain topological groups, and to approximately finite dimensional von Neumann algebras.
\end{abstract}

\section{Introduction}

This paper continues a study, begun in [27], of extensions of a von Neumann algebra by a locally compact group (of automorphisms). While [27] considered a rather special kind of extension, namely the crossed product of a von Neumann algebra by a group of automorphisms and a centre-valued, unitary, 2 cocycle on the group (a topic also considered in [32]), this paper is concerned with a much more general kind of extension. At the same time, our extensions are intimately connected with properties which a homomorphism from a given group into the outer automorphism classes of a given von Neumann algebra might have; in particular, it is invaluable to know when such a homomorphism is split. This same problem arises from consideration of Connes

Communicated by H. Araki, December 6, 1977.

* Department of Mathematics, University of Oslo, Oslo.

Current address: Department of Mathematics, University of Oregon, Eugene, Oregon 97403, U.S.A.

1) Partially supported by the Norwegian Research Council. 
invariant $\chi$ for $\mathrm{II}_{1}$-factors [8]; for these reasons, the study of such homomorphisms occupies a large portion of the paper.

Briefly, an extension $\{\mathscr{N}, I, \pi\}$ of a von Neumann algebra $\mathscr{M}$ by a locally compact group $G$ consists of a von Neumann algebra $\mathscr{\Omega}$ which is generated by an isomorphic copy $I(\mathscr{M})$ of $\mathscr{M}$, and a Borel family $\{\pi(g), g \in G\}$ of unitaries normalizing $I(\mathscr{M})$ and forming a representation of $G$ modulo $I(\mathscr{M})$. This represents one way in which the terms $M, N$ in an exact sequence $1 \rightarrow M \rightarrow N \rightarrow$ $G \rightarrow 1$ of locally compact groups may be replaced by von Neumann algebras $\mathscr{M}, \mathfrak{N}$ respectively. Our work is thus closely related to that of [28], [23]; another (different) generalization of the exact sequence above is given in [13]. It should be noted that the analogy with group extensions is more than schematic; if $\rho$ is any (continuous unitary) representation of $N$ above, the von Neumann algebra $\rho(N)^{\prime \prime}$ is indeed an extension of $\rho(M)^{\prime \prime}$ by $G$.

One of the main aims of this paper is to generalize the extension theory of (topological) groups as found for example in [22] and [21] to the von Neumann algebra situation. This is for the most part routine (albeit sometimes technical) and accomplished in Section 3 and Section 4; there is however one notable lack, in that we have been unable to find an intrinsic construction of the "Baer product" of two extensions of $\mathscr{M}$ by $G$. It should also be noted that due to the abundance of unitaries in a von Neumann algebra (the unitary group is in particular divisible), the relevant (second) cohomology groups frequently vanish, so that in many cases a given extension $\{\Re, I, \pi\}$ is "strongly equivalent" to another $\left\{\mathcal{I}, I, \pi_{1}\right\}$ where $\pi_{1}$ is a representation of $G$ (or, at worst, a representation modulo the centre of $I(\mathscr{M})$ ).

Throughout the paper, all von Neumann algebras are to have separable preduals, and all groups $G$ to be locally compact and separable. The organization is as follows; in Section 2, we give two (unrelated) technical results vial for later investigation. If $\alpha: G \rightarrow \operatorname{Aut}(\mathscr{M})$ is a Borel map (not necessarily a homomorphism) and $w: G \times G \rightarrow \mathscr{M}$ a unitary $\alpha$-2-cocycle, then $w \otimes 1$ cobounds in $\mathscr{M} \otimes$ $\mathscr{B}\left(L^{2}(G)\right)$ with respect to $\alpha \otimes \iota$. If $G$ is countable and $\mathscr{M}=\int_{\Gamma}^{\oplus} \mathscr{M}(r) d \mu(\gamma)$ is the central decomposition of $\mathscr{M}$, we give a description of the automorphisms $\alpha_{g}: g \in G$ in terms of "fields of fibre isomorphisms" between the components algebras $\{\mathscr{H}(r), r \in \Gamma\}$; this description reduces to the "point realization" of [19] when $\mathscr{M}$ is abelian, and to the direct integral decomposition when each $\alpha_{g}$ fixes the centre of $\mathscr{M}$ pointwise.

In Section 3 we associate to each extension $\{\mathscr{l}, I, \pi\}$ of $\mathscr{M}$ by $G$ a $G$-kernel 
i.e. a pair $\{\mathscr{H}, \theta\}$ where $\theta$ is a homomorphism of $G$ into $\operatorname{Out}(\mathscr{H})=\operatorname{Aut}(\mathscr{H}) /$ Int $(\mathscr{M})$. Necessary and sufficient conditions are derived, in terms of a centrevalued unitary 3-cocycle on $G$, (the "obstruction"), for a given $G$-kernel to arise from an extension, and an intrinsic description of the group operations among 3-cocycles is given in terms of the corresponding kernels. In Section 4, split kernels, i.e. kernels $\{\mathscr{M}, \theta\}$ for which there is a Borel homomorphism $\alpha: G \rightarrow \operatorname{Aut}(\mathscr{H})$ lifting $\theta$, are studied.

Criteria are developed for a kernel to be split in terms of the corresponding obstruction. In particular, when $\mathscr{M}$ is properly infinite, or of pure type $\mathbb{I I}_{1}$ and $G$ is finite, a kernel is split if and only if its obstruction is trivial. In case $\mathscr{M}$ is type $I$, it is automatic that all kernels are split and hence all obstructions are trivial. Each extension $\{\Re, I, \pi\}$ of a split kernel is shown to be "strongly equivalent" to a "normalized" extension $\left\{\Re, I, \pi_{1}\right\}$ where $\pi_{1}$ is a representation of $G$ modulo the centre of $I(\mathscr{H})$, or, in case $\mathscr{M}$ is properly infinite, or $\mathscr{M}$ of pure type $I I_{1}$ and $G$ finite, where $\pi_{1}$ is a representation of $G$. The algebraic structure of an extension of a split kernel is thus seen to be identical with that of a twisted crossed product. In Section 5 we give conditions on a (regularized) extension $\{\mathscr{l}, I, \pi\}$ of $\mathscr{M}$ by $G$ which ensure that it is a crossed product; these are in terms of the existence of a single pair of Plancherel (or dual) weights, one on $\mathscr{M}$ and one on $\mathscr{l}$. In case $G$ is countable and acts freely on $\mathscr{M}$ these are effectively the more familiar criteria (see e.g. [3], [12]) in terms of the existence of an expectation from $\mathscr{\eta}$ to $I(\mathscr{M})$. If the group $G$ is abelian we give an alternate characterization, in the spirit of [18], in terms of the existence of an appropriate "dual action" of the dual group of $G$ on $\Re$.

The final section presents applications; in particular we see that if a group $N$ is an extension of a connected group by an amenable group, then any representation of $N$ generates an approximately finite dimensional von Neumann algebra (see [7]). Also, in the case of properly infinite algebras $\mathscr{M}$, any twisted crossed product $\mathscr{N}$ of $\mathscr{M}$ by $G$ is in fact already an ordinary crossed product (perhaps with respect to a different action of $G$ ); this result applies also to certain finite groups and $I I_{1}$-algebras. Interest in twisted crossed products as a means of producing new von Neumann algebras is thus essentially reduced to the case of abelian algebras (see also [15] for a different approach to this problem); cohomological techniques seem insufficient to give any conclusive results in this case so that we do not attempt any classification of the von Neumann algebras arising in this way.

The author would like to express his debt to $A$. Connes, without whom 
this work would never have been begun.

\section{$\S 1$. Notations and Conventions}

Throughout, all von Neumann algebras $\mathscr{M}$ will be assumed to have separable preduals $\mathscr{M}_{*}$, and all Hilbert spaces are separable; when necessary, $\mathscr{M}$ will be considered as a Borel space with Borel structure generated by the strong *-topology. $\operatorname{Aut}(\mathscr{M})$ will denote the group of $*$-automorphisms of $\mathscr{M}$ with the topology of pointwise norm convergence against $\mathscr{M}_{*}$; this topology is Polish, so that the associated Borel structure is standard ([4], [14]). $\operatorname{Int}(\mathscr{M})$ denotes the normal subgroup of inner automorphisms, i.e. automorphisms of the form Ad $u, u$ unitary in $\mathscr{M}$, where $\operatorname{Ad} u(x)=u x u^{*}$. $\varepsilon$ denotes the quotient map $\varepsilon: \operatorname{Aut}(\mathscr{M}) \rightarrow \operatorname{Aut}(\mathscr{M}) / \operatorname{Int}(\mathscr{M})=\operatorname{Out}(\mathscr{M})$. By $\mathcal{U}(\mathscr{M})$, we mean the unitary group of $\mathscr{M}$, and $\mathscr{Z}(\mathscr{M})$ denotes the centre of $\mathscr{M}$; thus $\mathcal{U}(\mathscr{Z}(\mathscr{M})$ ) is the unitary group of the centre of $\mathscr{M}$. Whenever we consider the central decomposition $\mathscr{H}=\int_{\Gamma}^{\oplus} \mathscr{M}(r) d \mu(\gamma)$ we shall assume (as we may) that $\Gamma$ is a standard Borel space and $\mu$ a Borel measure on $\Gamma$ with $L^{\infty}(\Gamma, \mu)$ isomorphic with $\mathscr{Z}(\mathscr{M})$.

We will use $G$ to denote a locally compact, separable, toplogical group; we use (almost) invariably a left invariant Haar measure $d g$ on $G$. If $\mathscr{H}$ is a Hilbert space and $G$ is as above, $L^{2}(G ; \mathcal{H})$ denotes the Hilbert space of (equivalence classes of) measurable maps $\xi: G \rightarrow \mathscr{H}$ with $\int_{G}\|\xi(g)\|^{2} d g<\infty$, with inner product $\langle\xi, \eta\rangle=\int_{G}\langle\xi(g), \eta(g)\rangle d g$. When appropriate we identify $L^{2}(G ; \mathcal{H})$ with $L^{2}(G) \otimes \mathscr{H}$. Similarly if $\mathscr{N}$ is a von Neumann algebra, $L^{\infty}(G ; \mathscr{M})$ is the von Neumann algebra of (equivalence classes of) norm bounded measurable maps from $G$ to $\mathscr{M}$; it is canonically isomorphic with $L^{\infty}(G) \otimes \mathscr{M}$.

We refer to [22] (see also [21]) for the cohomology of locally compact groups. Briefly, if $A$ is a Polish abelian group and $g \in G \rightarrow \alpha_{g} \in \operatorname{Aut}(A)$ a representation of $G$ with $(g, a) \rightarrow \alpha_{g}(a)$ continuous on $G \times A$, we may consider the group (with pointwise operations) of all Borel maps $\omega: G \times \cdots \times G$ ( $n$ copies $) \rightarrow A$ with $\omega\left(g_{1}, \cdots, g_{n}\right)=0$ if any of $g_{1}, \cdots, g_{n}$ is the identity $e$ of $G$. Such a map $\omega$ is termed a Borel $n$-cochain, and we write $\omega \in C^{n}(G ; A)$.

Define maps $\partial: C^{n}(G ; A) \rightarrow C^{n+1}(G ; A)$ by

$$
\begin{gathered}
(\partial \omega)\left(g_{1}, \cdots, g_{n+1}\right)=\alpha_{g_{1}}\left(\omega\left(g_{2}, \cdots, g_{n+1}\right)\right)+\sum_{j=1}^{n}(-1)^{j} \omega\left(g_{1}, \cdots, g_{j} g_{j+1}, \cdots, g_{n+1}\right) \\
+(-1)^{n+1} \omega\left(g_{1}, \cdots, g_{n}\right)
\end{gathered}
$$


(we write $G$ multiplicatively and $A$ additively). If $\partial \omega=0$ we say $\omega$ is an $\alpha$ $n$-cocycle and write $\omega \in Z_{a}^{n}(G ; A)$; if $\omega$ is of the form $\partial \nu$ for $\nu \in C^{n-1}(G ; A)$ we say $\omega$ is an $\alpha$-n-coboundary and write $\omega \in B_{\alpha(}^{n}(G ; A)$. Since $\partial \circ \partial=0$ we have $B_{\alpha}^{n}(G ; A) \subseteq Z_{\alpha i}^{n}(G ; A)$; the $n^{t / h}$ cohomology group $H_{\alpha}^{n}(G ; A)$ of $G$ with coefficients in $A$ is the quotient $Z_{\alpha}^{n}(G ; A) / B_{a}^{n}(G ; A)$. By convention, $H_{\alpha}^{0}(G ; A)=$ $\left\{a \in A: \alpha_{g}(a)=a\right.$ for all $\left.g \in G\right\}$. Invariably, for us, $A$ will be of the form $\mathcal{U}(\mathscr{Z}(\mathscr{M})$ ) for some von Neumann algebra $\mathscr{M}$; if $\mathscr{M}$ is a factor, we will thus be considering the groups $H_{a}^{n}(G ; \mathrm{T})$ where $\mathbb{T}$ denotes the circle group.

\section{§2. Technical Preliminaries}

\section{2. 且. Non-abelian Cohomology}

We adopt the following convention; if $J=\left\{j_{1}, \cdots, j_{n}\right\}$ is a finite set of integers with $j_{1}<j_{2}<\cdots<j_{n}$, and if $a_{j}, j \in J$ are elements of a (possibly nonabelian) group, then $\prod_{j \in J} a_{i}$ denotes the element $a_{j_{1}} a_{j_{2}} \cdots a_{j_{n}}$.

Definition 2.1.1. Let $\alpha: g \in G \rightarrow \alpha_{g} \in \operatorname{Aut}(\mathscr{M})$ be an arbitrary Borel map. $A$ (unitary) $\alpha$-n-cocycle is then a Borel map $w: G \times G \times \cdots \times G$ ( $n$ copies) $\rightarrow$ $\mathcal{U}(\mathscr{M})$ satisfying

(a) $w\left(g_{1}, \cdots, g_{n}\right)=1$ if $g_{j}=e$ for some $j, 1 \leq j \leq n$,

(b) $\underset{\substack{j, 0 d d \\ 1 \leq j \leq n}}{\prod_{1}} w\left(g_{1}, \cdots, g_{j} g_{j+1}, \cdots, g_{n+1}\right) w\left(g_{1}, \cdots, g_{n}\right)^{(-1)^{n}}$

$$
=\alpha_{g_{1}}\left(w\left(g_{2}, \cdots, g_{n+1}\right)\right) \prod_{\substack{j, \text { even } \\ 1 \leq j \leq n}} w\left(g_{1}, \cdots, g_{j} g_{j+1}, \cdots, g_{n+1}\right) .
$$

for every $n+1$ elements $g_{1}, \cdots, g_{n+1}$ of $G$.

Definition 2.1.2. An $\alpha-n$-cocycle $w$ is an $\alpha$-coboundary if there is a Borel map $v: G \times G \times \cdots \times G \rightarrow \mathcal{U}(\mathscr{M})(n-1$ copies of $G)$ with $w=\partial v$ where

$$
\begin{aligned}
(\partial v)\left(g_{1}, \cdots, g_{n}\right)= & \alpha_{g_{1}}\left(v\left(g_{2}, \cdots, g_{n}\right)\right) \prod_{\substack{j, \text { even } \\
1 \leq j \leq n-1}} v\left(g_{1}, \cdots, g_{j} g_{j+1}, \cdots, g_{n}\right) \\
& \left(\prod_{\substack{j, \text { odd } \\
1 \leq j \leq n-1}} v\left(g_{1}, \cdots, g_{j} g_{j+1}, \cdots, g_{n}\right) v\left(g_{1}, \cdots, g_{n-1}\right)^{(-1)^{n-1}}\right)^{-1}
\end{aligned}
$$

Remark. The order of the terms in definitions 2.1.1 and 2.1.2 are of course adapted to each other; the results we prove remain true with other reasonable and mutually adapted definitions of cocycle and coboundary. Also, in general, $\partial$ as defined in 2.1.2 does not satisfy $\partial^{2}=1$. However if $g \rightarrow \alpha_{g}$ is 
a homomorphism and $w, v$ of 2.1.1 and 2.1.2 take values in an abelian subalgebra of $\mathscr{M}$ invariant under the $\alpha_{g}$, our definitions are the usual ones for (normalized) cocycles and coboundaries.

We begin with a result which seems special, in full generality, to the twodimensional situation.

Proposition 2.1.3. Let $g \in G \rightarrow \alpha_{g} \in \operatorname{Aut}(\mathscr{M})$ be an arbitrary Borel map, and w: $G \times G \rightarrow \mathcal{U}(\mathscr{M})$ an $\alpha$-2-cocycle. Consider $\beta_{g}=\alpha_{g} \otimes$ on $\mathscr{M} \otimes \mathscr{B}\left(L^{2}(G)\right)$. Then $(g, h) \in G \times G \rightarrow w(g, h) \otimes 1$ is a $\beta$-coboundary.

Proof. We first note that $u(g, h)=w(g, h) \otimes 1$ is clearly a $\beta$-2-cocycle; by assumption $w$ satisfies $\alpha_{g}(w(h, k)) w(g, h k)=w(g, h) w(g h, k)$ for all $g, h, k \in G$. We must produce a Borel map $g \in G \rightarrow v(g) \in \mathcal{U}\left(\mathscr{M} \otimes \mathscr{B}\left(L^{2}(G)\right)\right)$ with $w(g, h) \otimes 1$ $=\beta_{g}(v(h)) v(g) v(g h)^{*}$.

We may suppose that $\mathscr{M}$ acts on the Hilbert space $\mathscr{H}$, and that $\mathscr{M} \otimes$ $\mathscr{B}\left(L^{2}(G)\right)$ is realized on $L^{2}(G ; \mathscr{H})$. For $\xi \in L^{2}(G ; \mathscr{H})$ set $(v(h) \xi)(k)=w(h, k)$ $\xi(h k)$. Trivially $h \rightarrow v(h)$ is Borel. Also

$$
\begin{aligned}
\left(\beta_{g}(v(h)) v(g) v(g h)^{* \xi}\right)(k) & =\alpha_{g}(w(h, k))\left(v(g) v(g h)^{* \xi}\right)(h k) \\
& =\alpha_{g}(w(h, k)) w(g, h k) w(g h, k)^{*} \xi(k) \\
& =w(g, h) \xi(k) \\
& =((w(g, h) \otimes 1) \xi(k) .
\end{aligned}
$$

Thus $(\partial v)(g, h)=w(g, h) \otimes 1$ as required.

Q.E.D.

Proposition 2.1.3 has the disadvantage that it may well happen that $\mathscr{M} \otimes$ $\mathscr{B}\left(L^{2}(G)\right)$ is properly infinite while $\mathscr{M}$ is finite; this situation may be remedied in one of two ways (neither of which is entirely satisfactory).

Proposition 2.1.4. Let $G$ be a discrete group with order $\neq 2, g \in G \rightarrow \alpha_{g} \in$ $\operatorname{Aut}(\mathscr{M})$ an arbitrary map and $\omega: G \times G \rightarrow \mathcal{U}(\mathcal{Z}(\mathscr{M}))$ an $\alpha$-2-cocycle. Then there is

i) a full $I_{1}$ factor $\mathscr{Q}$, and a map $\beta: g \in G \rightarrow \beta_{g} \in \operatorname{Aut}(\mathscr{M} \otimes \mathscr{Q})$ with $\beta_{g}(x \otimes 1)=\alpha_{g}(x) \otimes 1, g \in G, x \in \mathscr{M}$,

ii) unitaries $u(g), g \in G$, generating $\mathscr{L}$, such that $w(g, h) \otimes 1=\beta_{g}(1 \otimes u(h))$ $\left(1 \otimes u(g) u(g h)^{*}\right)$.

Further, if $\alpha$ is a homomorphism, we may also choose $\beta$ to be a homomorphism.

Proof. We recall the following result from [25]; if $F$ is the free non-abelian 
group on generators $x_{1}, x_{2}, \cdots, x_{n}, n=2,3, \cdots \infty$, and $\left\{\lambda_{j}, j=1, \cdots, n\right\}$ a given set of complex numbers with $\left|\lambda_{j}\right|=1 j=1,2, \cdots, n$, then there is an automorphism $\delta$ of the algebra generated by the left regular representation $\lambda^{F}$ of $F$ determined by $\delta\left(\lambda^{F}\left(x_{j}\right)\right)=\lambda_{j} \lambda^{F}\left(x_{j}\right), j=1,2, \cdots, n$.

Let $\Phi_{G}$ denote the free non-abelian group on the symbols $x_{g}, g \in G-\{e\}$. We shall take $\mathscr{Q}$ to be the algebra generated by the left regular representation $\lambda$ of $\Phi_{G}$, and $u(g)=\lambda\left(x_{g}\right)$.

First, consider the endomorphisms $\widetilde{\delta}_{g}, g \in G$, of $\Phi_{G}$ determined by $\widetilde{\delta}_{g}\left(x_{h}\right)$ $=x_{g h} x_{g}^{-1}$. We easily see that $\widetilde{\delta}_{g} \widetilde{\delta}_{h}\left(x_{k}\right)=\widetilde{\delta}_{g}\left(x_{h k} x_{h}^{-1}\right)=x_{g h k} x_{g}^{-1} x_{g} x_{g h}^{-1}=x_{g h k} x_{g h}^{-1}=$ $\widetilde{\delta}_{g h}\left(x_{k}\right)$, so that each $\widetilde{\delta}_{g}$ is in fact an automorphism, and $g \rightarrow \widetilde{\delta}_{g}$ a representation of $G$ as automorphisms of $\Phi_{G}$.

Let $\mathscr{M}=\int_{\Gamma}^{\oplus} \mathscr{M}(r) d \mu(\gamma)$ be the central decomposition of $\mathscr{M}$, and $r \rightarrow$ $\omega_{\gamma}(g, h)$ a fixed representation of $\omega(g, h) \in \mathcal{U}(\mathscr{Z}(\mathscr{M}))$ as a Borel function on $\Gamma$ with values in the circle. In view of the fact that each automorphism of $\Phi_{G}$ lifts to an automorphism of $\mathscr{L}$, and by virtue of the result of [25] quoted above, we may, for each $r \in \Gamma$ construct automorphisms $\delta_{g}^{\gamma}$ of $\mathscr{Q}$ characterized by $\delta_{g}^{\gamma}(u(h))=\omega_{\gamma}(g, h) u(g h) u(g)^{*}$. If we identify $\mathscr{M} \otimes \mathscr{Q}$ with $\int_{\Gamma}^{\oplus} \mathscr{M}(\gamma) \otimes \mathscr{Q} d \mu(\gamma)$ we may thus define automorphisms $\delta_{g}, g \in G$ of $\mathscr{M} \otimes \mathscr{Q}$ by $\delta_{g}=\int_{\Gamma}^{\oplus} \iota \otimes \delta_{g}^{\gamma} d \mu(\gamma)-$ it is clear that for each $g$, the field of automorphisms $\gamma \rightarrow \iota \otimes \delta_{g}^{\gamma} \in \operatorname{Aut}(\mathscr{M}(\gamma) \otimes \mathscr{Q})$ is measurable (see [26]).

By construction $\delta_{g}(x \otimes 1)=x \otimes 1, \quad x \in \mathcal{M}, \quad$ and $\quad \delta_{g}(1 \otimes u(h))=\omega(g, h) \otimes$ $u(g h) u(g)^{*}$. Set $\beta_{g}=\delta_{g} \circ\left(\alpha_{g} \otimes \iota\right)$, so that $\beta_{g}$ and $\alpha_{g}$ agree on $\mathscr{M} \otimes 1$; evidently we still have $\beta_{g}(1 \otimes u(h))=\omega(g, h) \otimes u(g h) u(g)^{*}$, i.e. $\omega(g, h) \otimes 1=\beta_{g}(1 \otimes u(h))$ $\left(1 \otimes u(g) u(g h)^{*}\right)$.

Finally, if $g \rightarrow \alpha_{g}$ is a homomorphism, then so is $g \rightarrow$ the restriction of $\beta_{g}$ to $M \otimes 1$. On the other hand, we always have

$$
\begin{aligned}
\beta_{g} \circ \beta_{h}(1 \otimes u(k)) & =\beta_{g}\left(\omega(h, k) \otimes u(h k) u(h)^{*}\right) \\
& =\alpha_{g}(\omega(h, k)) \omega(g, h k) \omega(g, h)^{*} \otimes u(g h k) u(g)^{*} u(g) u(g h)^{*} \\
& =\omega(g h, k) \otimes u(g h k) u(g h)^{*} \\
& =\beta_{g h}(1 \otimes u(k))
\end{aligned}
$$

so that $\beta_{g} \circ \beta_{h}=\beta_{g h}$ on $1 \otimes \mathscr{Q}$.

Q.E.D.

Remarks. 1) In 2.1.4, if $g \rightarrow \varepsilon \circ \alpha_{g} \in \operatorname{Out}(\mathscr{M})$ is a homomorphism, so is $g \rightarrow \varepsilon \circ \beta_{g} \in \operatorname{Out}(\mathscr{M} \otimes \mathscr{Q})$. 
2) In the proof of 2.1.4, we may replace $\Phi_{G}$ by $A_{G}$, the free abelian group on the generators $x_{g}, g \in G-\{e\}$. The construction may be repeated verbatim, with $\mathscr{Q}$ being replaced by the (abelian) algebra generated by the left regular representation of $A_{G}$.

Our final result seems to be valid only when we are dealing with representations of groups as automorphisms.

Proposition 2.1.5. Let $g \in G \rightarrow \alpha_{g} \in \operatorname{Aut}(\mathscr{M})$ be a Borel homomorphism and $\lambda$ the left regular representation of $G$ on $L^{2}(G)$. Set $\beta_{g}=\alpha_{g} \otimes \operatorname{Ad} \lambda(g)$, on $\mathscr{M} \otimes L^{\infty}(G)$. Then if $w$ is any $\alpha$ - $n$-cocycle $n \geq 1,\left(g_{1}, \cdots, g_{n}\right) \rightarrow w\left(g_{1}, \cdots, g_{n}\right) \otimes 1 \in$ $\mathscr{M} \otimes L^{\infty}(G)$ is a $\beta$-coboundary.

Proof. We may suppose $\mathscr{M}$ acts on $\mathscr{H}$, and that $\mathscr{M} \otimes L^{\infty}(G)$ (which we may identify with $L^{\infty}(G ; \mathscr{M})$ ) is realized on $L^{2}(G ; \mathscr{H})$.

For $g_{1}, \cdots, g_{n-1} \in G$, and $\xi \in L^{2}(G ; \mathscr{H})$, set

$$
\left(v\left(g_{1}, \cdots, g_{n-1}\right) \xi\right)(k)=\alpha_{k}\left(w\left(k^{-1}, g_{1}, \cdots, g_{n-1}\right)\right) \xi(k) .
$$

Clearly $v\left(g_{1}, \cdots, g_{n-1}\right) \in \mathcal{U}\left(L^{\infty}(G ; \mathscr{M})\right)$ and $\left(g_{1}, \cdots, g_{n-1}\right) \rightarrow v\left(g_{1}, \cdots, g_{n-1}\right)$ is a Borel map; also $v\left(g_{1}, \cdots, g_{n-1}\right)=1$ if any of $g_{1}, \cdots, g_{n-1}$ is the identity. For each $g \in G$, we let $W(g)$ be a unitary on $\mathscr{H}$ with $\operatorname{Ad} W(g)=\alpha_{g}$; (such unitaries exist by virtue of [14]). We now compute

$$
\begin{aligned}
\left(\beta_{g_{1}}\left(v\left(g_{2}, \cdots, g_{n}\right)\right) \xi\right)(k) & =W\left(g_{1}\right)\left(v\left(g_{2}, \cdots, g_{n}\right)\left(W\left(g_{1}\right)^{*} \otimes \lambda\left(g_{1}\right)^{*}\right) \xi\right)\left(g_{1}^{-1} k\right) \\
& =W\left(g_{1}\right) \alpha_{g_{1}^{-1} k}\left(w\left(k^{-1} g_{1}, g_{2}, \cdots, g_{n}\right)\right) W\left(g_{1}\right)^{*} \xi(k) \\
& =\alpha_{k}\left(w\left(k^{-1} g_{1}, g_{2}, \cdots, g_{n}\right)\right) \xi(k) .
\end{aligned}
$$

Using the cocycle equation 2.1.1. (b) with the $n+1$ variables $k^{-1}, g_{1}, \cdots, g_{n}$ it is now trivial to verify $(\partial v)\left(g_{1}, \cdots, g_{n}\right)=w\left(g_{1}, \cdots, g_{n}\right) \otimes 1$ as required. Q.E.D.

It should be noted that in the situation of $2.1 .5,\left(g_{1}, \cdots, g_{n}\right) \rightarrow w\left(g_{1}, \cdots, g_{n}\right) \otimes 1$ cobounds in $\mathscr{M} \otimes \mathscr{B}\left(L^{2}(G)\right)$ with respect to $\beta_{g}=\alpha_{g} \otimes \operatorname{Ad} \lambda(g)$; of course even if $w$ takes values in $\mathscr{Z}(\mathscr{M})$, the cochain $v$ constructed above need not take values in the centre of $\mathscr{M} \otimes \mathscr{B}\left(L^{2}(G)\right)$. The result of 2.1 .5 has been used implicity in the literature; we note in particular $[3 ; 1.2 .4]$; the last result plays a role in [9] (in the one dimensional context).

\subsection{Automorphisms of Non-factors}

Let $\mathscr{M}$ be a von Neumann algebra with central decomposition $\mathscr{M} \simeq$ 
$\int_{\Gamma}^{\oplus} \mathscr{H}(\gamma) d \mu(\gamma)$, and let $\alpha \in \operatorname{Aut}(\mathscr{M})$, we are interested in ways of describing $\alpha$ in terms of the component algebras $\{\mathscr{M}(r), r \in \Gamma\}$. Unless $\alpha$ fixes the centre of $\mathscr{M}$ pointwise, $\alpha$ does not admit a direct integral decomposition; nevertheless, if $\mathscr{M}$ is abelian it is well known (see [19]) that $\alpha$ admits a "point realization" on $\Gamma$ (in this case $\mathscr{M} \simeq L^{\infty}(\Gamma$, $\mu)$ ) i.e. there is an invertible transformation $T$ on $\Gamma$ with $T \mu \sim \mu$ and $(\alpha f)(\gamma)=f\left(T^{-1} \gamma\right)$ a.e. for $f \in L^{\infty}(\Gamma, \mu)$. We shall establish here a simultaneous generalization of the point realization in the abelian case, and of the direct integral decomposition in the case $\alpha(z)=z, z \in$ $\mathscr{Z}(\mathscr{M})$.

We fix a central decomposition $\mathscr{M}=\int_{\Gamma}^{\oplus} \mathscr{H}(r) d \mu(\gamma)$, and $\alpha \in \operatorname{Aut}(\mathscr{M})$. We let $T$ be a point realization of the restriction of $\alpha$ to $\mathscr{Z}(\mathscr{M})$ on $\Gamma$. When $x \in \mathscr{M}$ has decomposition $x=\int_{\Gamma}^{\oplus} x(r) d \mu(\gamma)$, we write $x \sim x(r)$.

Proposition 2.2.1. With the above notations there is a T-invariant $\mu$-null set set $N \subseteq \Gamma$, and normal isomorphisms $\kappa_{\gamma}: \mathscr{H}(\gamma) \rightarrow \mathscr{H}(T \gamma), r \in \Gamma-N$, with

(i) $r \rightarrow x(\gamma) \in \mathscr{M}(\gamma)$ is Borel if and only if $\gamma \rightarrow \kappa_{\gamma}(x(\gamma)) \in \mathscr{M}(T \gamma)$ is Borel,

(ii) if $x \sim(x(\gamma))$ then $\alpha(x) \sim(y(\gamma))$ where $y(\gamma)=\kappa_{T^{-1} \gamma}\left(x\left(T^{-1} \gamma\right)\right) \mu$-a.e.

Proof. Let $\mathcal{A} \subseteq \mathscr{M}$ be a $\sigma$-weak*-dense, norm separable $C^{*}$-subalgebra; we may suppose $\mathfrak{A}=\mathscr{A} \cap \mathscr{Z}(\mathscr{M})$ is $\sigma$-weak*-dense in $\mathscr{Z}(\mathscr{M})$, and that $\mathcal{A}$ (and $\mathfrak{U})$ are $\alpha$-invariant. We may further suppose that $\Gamma$ is the spectrum of $\mathfrak{A}$.

Let $\pi$ denote the identity representation of $\mathcal{A}$ in the decomposable von Neumann algebra $\mathscr{M}$. From [10], $\pi$ admits a decomposition $\pi=\int_{\Gamma}^{\oplus} \pi_{\gamma} d \mu(\gamma)$; we may assume, after deletion of a null set that the $\pi_{\gamma}$ are representations and that $\left\{\pi_{\gamma}(\mathcal{A})\right\}^{\prime \prime}=\mathscr{M}(\gamma)$. Note also that the following diagram commutes

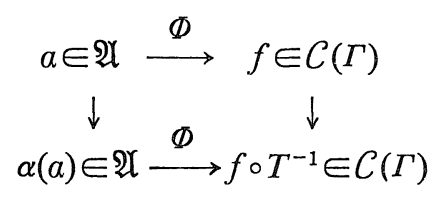

where $\Phi$ is the Gelfand representation of $\mathfrak{U}$ on its spectrum. In view of the fact that we may assume the automorphism $\alpha$ is implemented by some unitary on the Hilbert space of $\mathscr{M}$, it follows from the proofs of existence and uniqueness of decompositions of representations of $C^{*}$-algebras (see [10], especially 8.2.4) that for almost all $\gamma \in \Gamma$ the representations $\pi_{\gamma}$ and $\pi_{T \gamma^{\circ} \alpha}$ are unitarily equivalent. So for $r \in \Gamma-N$, where $N$ is $\mu$-null and $T$-invariant, there are unique 
isomorphisms $\kappa_{\gamma}: \mathscr{M}(\gamma) \rightarrow \mathscr{M}(T \gamma)$ with $\kappa_{\gamma}\left(\pi_{\gamma}(x)\right)=\pi_{T \gamma}(\alpha(x)), x \in \mathcal{A}$. The claims of the Proposition now follow, as $\left\{\pi_{\gamma}(\mathcal{A} A)\right\}^{\prime \prime}=\mathscr{M}(\gamma), \gamma \in \Gamma$, and for $x \in \mathcal{A}$, $x \sim\left(\pi_{\gamma}(x)\right)$.

Q.E.D.

The following generalization is immediate.

Proposition 2.2.2. Let $G$ be a countable group, and $\left\{\alpha_{g}, g \in G\right\}$ a set of automorphisms of $\mathscr{M}$ such that $g \in G \rightarrow \theta_{g}=$ restriction of $\alpha_{g}$ to $\mathscr{Z}(\mathscr{M})$ is a homomorphism. Let $\mathscr{M}=\int_{\Gamma}^{\oplus} \mathscr{M}(\gamma) d \mu(\gamma)$ be the central decomposition, and $(g, r) \in$ $G \times \Gamma \rightarrow g_{\gamma} \in \Gamma$ a point realization of $\theta$. Then, for $\gamma \in \Gamma-N$, where $N$ is $\mu-n u l l$ and G-invariant, there are normal isomorphisms $\kappa_{(g, \gamma)}: \mathscr{M}(\gamma) \rightarrow \mathscr{M}(g \gamma)$ with

(i) $r \rightarrow x(\gamma) \in \mathscr{M}(\gamma)$ Borel if and only if $r \rightarrow \kappa_{(g, \gamma)}(x(\gamma)) \in \mathscr{M}(g r)$ is Borel for each $g \in G$;

(ii) if $x \sim(x(\gamma))$, then $\alpha_{g}(x) \sim(y(\gamma))$ where $y(\gamma)=\kappa_{\left(g, g^{-1} \gamma\right)}\left(x\left(g^{-1} \gamma\right)\right) \mu-$ a.e. If, in addition, $g \rightarrow \alpha_{g}$ is a homomorphism, we havc

(iii) $\kappa_{h, g \gamma)} \circ \kappa_{(g, \gamma)}=\kappa_{(h g, \gamma)}, g, h \in G, r \in \Gamma-N$.

We omit the obvious proof.

Remarks. (i) Proposition 2.2.2. probably remains valid if $g \rightarrow \alpha_{g}$ is a continuous representation of $G$ in $\operatorname{Aut}(\mathscr{M})$. However, the author has not been able to find a systematic method of deleting null sets to effect the proof.

(ii) In case $G$ is countable, and $g \rightarrow \alpha_{g}$ is a representation, 2.2.2 (iii) is saying that $(g, \gamma) \in G \times \Gamma \rightarrow \kappa_{(g, \gamma)}$ is a representation of the groupoid $G \times \Gamma$ (see [15]) as "fibre isomorphisms".

In some cases, 2.2.2. allows a description of $\operatorname{Aut}(\mathscr{M})$ by means of a (split) exact sequence. If $\mathscr{M}=\int_{\Gamma}^{\oplus} \mathscr{M}(\gamma) d \mu(\gamma)$ is the central decomposition, define an equivalence relation $\mathscr{R}_{\mathscr{M}}$ on $\Gamma$ by $\left(r, \gamma^{\prime}\right) \in \mathscr{R}_{\mathscr{M}}$ if and only if $\mathscr{M}(\gamma)$ and $\mathscr{M}\left(r^{\prime}\right)$ are algebraically isomorphic. By [11], equivalence classes under $\mathscr{R}_{\mathscr{M}}$ are Borel. Recall that $\mathscr{M}$ is said to be centrally smooth ([11], [24]) if there is a $\mu$-null Borel set $N \subseteq \Gamma$ such that the quotient $(\Gamma-N) \mid \mathscr{R}_{\mathscr{M}}$ is countably separated.

Definition 2.2.3. (i) $\operatorname{Aut}_{\mathscr{Z}}(\mathscr{M})$ is the (normal) subgroup of $\operatorname{Aut}(\mathscr{M})$ consisting of those automorphisms $\alpha$ with $\alpha(z)=z, z \in \mathscr{Z}(\mathscr{M})$.

(ii) Aut $\mathscr{M}(\Gamma)$ is the group of equivalence classes of measurable automorphisms $\varphi$ of $\Gamma$ with $\varphi \mu \sim \mu$ and $\left(\gamma, \varphi^{-1}(\gamma)\right) \in \mathscr{R}_{\mathscr{M}}$ for $\mu$-almost all $\gamma$, where we identify $\varphi_{1}$ and $\varphi_{2}$ if $\varphi_{1}(\gamma)=\varphi_{2}(\gamma) \mu$-a.e. (The product is composition.) 
Proposition 2.2.4. Let $\mathscr{M}$ be centrally smooth. Then there is a split exact sequence $1 \rightarrow \operatorname{Aut}_{\mathscr{L}}(\mathscr{M}) \rightarrow \operatorname{Aut}(\mathscr{M}) \rightarrow \operatorname{Aut}_{\mathscr{M}}(\Gamma) \rightarrow 1$.

Proof. We construct a multiplicative lifting of $\operatorname{Aut} \mathscr{M}(\Gamma)$ into $\operatorname{Aut}(\mathscr{M})$.

We may assume $(\Gamma, \mu)$ is a probability space, and that (after deletion of a null set) $\Gamma \mid \mathscr{R}_{\mathscr{M}}=\Omega$ is analytic; we let $\pi: \Gamma \rightarrow \Omega$ be the natural surjection and $\tilde{\mu}$ be the image of $\mu$ under $\pi$. Thus, in the decomposition $\mu=\int_{\Omega}^{\oplus} \mu_{\omega} d \tilde{\mu}(\omega)$ of $\mu$ with respect to $\pi$ (see [11]) we may assume the $\mu_{\omega}$ are probability measures, and $\mu_{\omega}\left(\Gamma-\pi^{-1}(\omega)\right)=0$ for all $\omega$. By the "partial integration" technique of [24], we have $\mathscr{M}=\int_{\Omega}^{\oplus} \mathscr{M}(\omega) d \mu(\omega)$ where $\mathscr{M}(\omega)=\int_{\Gamma}^{\oplus} \mathscr{M}(\gamma) d \mu_{\omega}(\gamma)$.

By standard section theorems (see e.g. [2]), we may choose a universally measurable map $s: \omega \in \Omega \rightarrow s(\omega) \in \pi^{-1}(\omega) \subset \Gamma$; thus for (almost all) $\omega \in \Omega, \mathscr{M}(\omega)$ is isomorphic with $\mathscr{M}(s(\omega)) \otimes L^{\infty}\left(\Gamma, \mu_{\omega}\right)$.

Let now $\varphi$ be an invertible measurable transformation on $\Gamma$ with $\varphi \mu \sim \mu$ and $(r, \varphi(\gamma)) \in \mathscr{R}_{\mathscr{M}}$ for all $r$ (we have deleted a null set from $\Gamma$ to effect this). Each such $\varphi$ gives rise to an automorphism $\alpha_{\omega}^{\varphi}$ of $\mathscr{M}(s(\omega)) \otimes L^{\infty}\left(\Gamma, \mu_{\omega}\right)$ via $\alpha_{\omega}^{\varphi}(x \otimes f)=x \otimes \beta_{\omega}^{\varphi}(f), x \in \mathscr{M}(s(\omega)), f \in L^{\infty}\left(\Gamma, \mu_{\omega}\right)$, where $\left(\beta_{\omega}^{\varphi} f\right)(\gamma)=f\left(\varphi^{-1}(r)\right)$. Since $\varphi$ is measurable, the field of automorphisms $\omega \rightarrow \alpha_{\omega}^{\varphi} \in \operatorname{Aut}(\mathscr{M}(\omega))$ is measurable as a field of automorphisms over $\omega \rightarrow \mathscr{M}(\omega)$ (see [26]). We set $\alpha^{\varphi}=\int_{\Omega}^{\oplus} \alpha_{\omega}^{\varphi} d \tilde{\mu}(\omega)$ on $\mathscr{M}$. Clearly, $\alpha^{\varphi}$ depends only on the equivalence class of $\varphi$ in $\operatorname{Aut}_{\mathscr{M}}(\Gamma)$, and $\alpha^{\varphi} \circ \alpha^{\psi}=\alpha^{\varphi \circ \psi}$.

It remains to show that any automorphism $\alpha \in \operatorname{Aut}(\mathscr{M})$ is of the form $\beta \circ \alpha^{\varphi}$ for some $\beta \in \operatorname{Aut}_{\mathscr{Z}}(\mathscr{M}), \varphi \in \operatorname{Aut}_{\mathscr{M}}(\Gamma)$ (since clearly $\alpha^{\varphi} \in \operatorname{Aut}_{\mathscr{L}}(\mathscr{M}) \mathrm{im}$ plies $\varphi=$ identity a.e.). But from the proof of 2.2.1, given $\alpha \in \operatorname{Aut}(\mathscr{M})$ there is a measurable map $\varphi$ on $\Gamma$ (written $T^{-1}$ in 2.2.1) and normal isomorphisms $\kappa_{\gamma}: \mathscr{M}(\gamma) \rightarrow \mathscr{M}\left(\varphi^{-1}(\gamma)\right)$ with $(\alpha(x))(\gamma)=\kappa_{\varphi(\gamma)}(x(\varphi(\gamma))$, $\mu$-a.e. for $x \in \mathscr{M}$. Thus $\varphi \in \operatorname{Aut}_{\mathscr{M}}(\Gamma)$ and for $x \in \mathscr{M}$,

$$
\begin{aligned}
\left(\alpha \circ \alpha^{\varphi}(x)\right)(\gamma) & =\kappa_{(\varphi) \gamma}\left(\left(\alpha^{\varphi}(x)\right)(\varphi(\gamma))\right) & & \mu \text {-a.e. } \\
& =\kappa_{\varphi(\gamma) \circ \iota_{\gamma}^{\varphi}(\gamma)}(x(\gamma)) & & \mu \text {-a.e. }
\end{aligned}
$$

where $\iota_{\gamma}^{\varphi}(\gamma): \mathscr{H}(\gamma) \rightarrow \mathscr{M}(\varphi(\gamma))$ is the identification of $\mathscr{H}(\gamma)$ with $\mathscr{M}(\varphi(\gamma))$ used above (in e.g. the isomorphism $\mathscr{M}(\omega)=\mathscr{H}(s(\omega)) \otimes L^{\infty}\left(\Gamma, \mu_{\omega}\right)$ ). But $\kappa_{\varphi(\gamma)} \circ \iota_{\gamma}^{\varphi(\gamma)}$ is an automorphism of $\mathscr{H}(r)$; thus $\alpha \circ \alpha^{\varphi}$ is expressed as a direct integral of automorphisms of $\mathscr{M}(r)$, so that $\alpha \circ \alpha^{\varphi} \in \operatorname{Aut}_{\mathscr{L}}(\mathscr{M})$.

Q.E.D.

Remark. In an obvious (but undefined) sense $\operatorname{Aut} \mathscr{L}(\mathscr{M})=\int_{\Gamma}^{\oplus} \operatorname{Aut}(\mathscr{M}(\gamma)$ ) 
$d \mu(\gamma)$ and the above exact sequence then reads

$$
1 \rightarrow \int_{\Gamma}^{\oplus} \operatorname{Aut}(\mathscr{M}(r)) d \mu(r) \rightarrow \operatorname{Aut}\left(\int_{\Gamma}^{\oplus} \mathscr{M}(r) d \mu(r)\right) \rightarrow \operatorname{Aut} \mathscr{M}_{M}(\Gamma) \rightarrow 1 .
$$

\section{§3. Kernels}

\subsection{Kernels and Extensions}

We begin with definitions; as usual, von Neumann algebras and groups are supposed to be separable.

Definition 3.1.1. A $G$-kernel $\{\mathscr{M}, \theta\}$ consists of

(i) a von Neumann algebra $\mathscr{M}$,

(ii) a homomorphism $\theta: g \in G \rightarrow \operatorname{Out}(\mathscr{M})$ which admits a Borel lifting to $\operatorname{Aut}(\mathscr{M})$ i.e. for which there is a Borel map $\alpha: G \rightarrow \operatorname{Aut}(\mathscr{M})$ with $\varepsilon \circ \alpha=\theta$.

We do not assume here the map $\alpha$ of 3.1.1 (ii) is a homomorphism; this situation is discussed in Section 4. The assumption that $\theta$ admits a Borel lifting seems necessary in view of the fact that $\operatorname{Int}(\mathscr{M})$ may fail to be closed in $\operatorname{Aut}(\mathscr{M})$; however, when $\operatorname{Int}(\mathscr{M})$ is closed (i.e. $\mathscr{M}$ is full, [4]) (ii) above is easily seen to be equivalent to requiring $\theta$ to be continuous (cf. [22]).

Definition 3.1.2. An extension $\{\mathscr{l}, I, \pi\}$ of a von Neumann algebra $\mathscr{M}$ by a group $G$ consists of

(i) a von Neumann algebra $\Re$, and an isomorphism $I$ of $\mathscr{M}$ into $\mathscr{\eta}$;

(ii) a Borel map $\pi: g \in G \rightarrow \pi(g) \in \mathcal{U}(\mathcal{I})$ satisfying

(a) $\pi(g) I(\mathscr{M}) \pi(g)^{*}=I(\mathscr{M})$

(b) $\pi(g) \pi(h) \in I(\mathscr{M}) \pi(g h)$

(c) $\quad \Re=\{I(\mathscr{M}) \cup\{\pi(g): g \in G\}\}^{\prime \prime}$

Here, we are concerned with the relationships between $G$-kernels and extensions of $\mathscr{M}$ by $G$, paralleling a similar relationship in the theory of group extensions. There are two prime sources of examples of extensions.

Example 3.1.3. Let $\{\mathscr{M}, G, \alpha, \omega\}$ be a projective covariant system so that $\alpha$ is a representation of $G$ in $\operatorname{Aut}(\mathscr{M})$ and $\omega \in Z_{\alpha}^{2}(G ; \mathcal{U}(\mathscr{L}(\mathscr{M}))$ ). If $\mathscr{M}$ acts on the Hilbert space $\mathcal{H}$, we may define operators on $L^{2}(G ; \mathscr{H})$ by

$$
\begin{aligned}
& \left(I^{\alpha}(x) \xi\right)(g)=\alpha_{g}^{-1}(x) \xi(g), x \in \mathscr{M}, \\
& \left(\lambda^{\omega}(h) \xi\right)(g)=\omega\left(g^{-1}, h\right) \xi\left(h^{-1} g\right), h \in G
\end{aligned}
$$

where $\xi \in L^{2}(G ; \mathscr{H})$. We set $\mathscr{R}=\mathscr{R}(\mathscr{M}, G, \alpha, \omega)=\left\{I^{\alpha}(x), x \in \mathscr{M}, \lambda^{\omega}(g), g \in G\right\}^{\prime \prime}$. 
In this case

$$
\begin{aligned}
& \lambda^{\omega}(g) I^{\omega}(x) \lambda^{\omega}(g)^{*}=I^{\omega}\left(\alpha_{g}(x)\right), x \in \mathcal{M}, g \in G, \\
& \lambda^{\omega}(g) \lambda^{\omega}(h)=I^{\alpha}(\omega(g, h)) \lambda^{\omega}(g h), \quad g, h \in G,
\end{aligned}
$$

so that $\left\{\Re, I^{\alpha}, \lambda^{\omega}\right\}$ is indeed an extension of $\mathscr{M}$ by $G$. This of course is the twisted crossed product studied in [27] and [32].

Example 3.1.4. Let $1 \rightarrow M \rightarrow N \rightarrow G \rightarrow 1$ be any exact sequence of locally compact separable topological groups (see [22]), and let $\rho$ by any continuous unitary representation (or even projective representation) of $N$ on a separable Hilbert space; let $\mathscr{M}=\rho(M)^{\prime \prime}, \mathscr{I}=\rho(N)^{\prime \prime}$ and $I: \mathscr{M} \rightarrow \mathscr{N}$ the inclusion map. If $g \in G \rightarrow n_{g} \in N$ is any Borel right inverse to the surjection $N \rightarrow G$, and $\pi(g)$ $=\rho\left(n_{g}\right)$, then $\{\mathscr{R}, I, \pi\}$ is an extension of $\mathscr{M}$ by $G$.

There is one obvious relationship between extensions and kernels; if $\{\mathscr{N}, I, \pi\}$ is an extension of $\mathscr{M}$ by $G$, and $\alpha_{g}=I^{-1} \circ \operatorname{Ad} \pi(g) \circ I \in \operatorname{Aut}(\mathscr{M})$, then, from 3.1.2 (b), $g \rightarrow \theta_{g}=\varepsilon\left(\alpha_{g}\right) \in \operatorname{Out}(\mathscr{M})$ is a homomorphism. Clearly $\{\mathscr{M}, \theta\}$ is then a $G$-kernel. In this situation, we say that the extension $\{\mathscr{I}, I, \pi\}$ realizes or extends the kernel $\{\mathscr{M}, \theta\}$, and that $\{\mathscr{M}, \theta\}$ is associated to the extension. An extendable kernel is one which is realized by some extension.

As we shall see, not all kernels are extendable; our immediate goal is to determine those which are.

Let $\{\mathscr{M}, \theta\}$ be a given $G$-kernel, and $g \rightarrow \alpha_{g} \in \operatorname{Aut}(\mathscr{M})$ a Borel map with $\varepsilon \circ \alpha=\theta$. Since $\theta_{g} \circ \theta_{h}=\theta_{g h}, g, h \in G$ we may choose for each $g, h \in G, v(g, h) \in$ $\vartheta(\mathscr{H})$ with $\alpha_{g} \circ \alpha_{h}=\operatorname{Adv}(g, h) \circ \alpha_{g h}$. Since $\operatorname{Int}(\mathscr{M})$ is isomorphic with $U(\mathscr{M}) /$ $\mathcal{U}(\mathscr{Z}(\mathscr{M})$ ), and $U(\mathscr{Z}(\mathscr{M})$ ) is closed in $U(\mathscr{M})$ (with respect to the strong-* topology), we may by standard section theorems assume $(g, h) \in G \times G \rightarrow v(g, h) \in$ $\mathcal{U}(\mathscr{M})$ is Borel. Comparing $\alpha_{g} \circ\left(\alpha_{h} \circ \alpha_{k}\right)$ with $\left(\alpha_{g} \circ \alpha_{h}\right) \circ \alpha_{k}$ we see $\operatorname{Ad} \alpha_{g}(v(h, k))$ $\times v(g, h k)=\operatorname{Ad} v(g, h) v(g h, k)$ for $g, h, k \in G$. There is thus a Borel function $f:$ $G \times G \times G \rightarrow \mathcal{U}(\mathcal{Z}(\mathscr{M}))$ determined by $\alpha_{g}(v(h, k)) v(g, h k)=f(g, h, k) v(g, h) v(g h, k)$ for all $g, h, k \in G$. Since we may choose the maps $\alpha, v$ so that $\alpha_{e}=$ identity and $v(g, h)=1$ if $g$ or $h$ is the identity, we see $f(g, h, k)=1$ if any of $g, h$ or $k$ is the identity. We note also that the restriction of the automorphisms $\alpha_{g}$ to $\mathscr{L}(\mathscr{M})$ defines a representation of $G$ in $\operatorname{Aut}(\mathscr{Z}(\mathscr{M}))$ which depends only on $\theta$; we denote this action by $\theta$ also.

Lemma 3.1.5. Let $\{\mathscr{M}, \theta\}$ and $f: G \times G \times G \rightarrow \mathcal{U}(\mathscr{Z}(\mathscr{M}))$ be as above. Then 
(i) $f \in Z_{\theta}^{3}(G ; \mathcal{U}(\mathscr{Z}(\mathscr{M})))$

(ii) the cohomology class of $f$ in $H_{\theta}^{3}(G ; \mathcal{U}(\mathcal{Z}(\mathscr{M})))$ is independent of the choices $\alpha, v$ made above, and depends only on $\{\mathscr{H}, \theta\}$.

(iii) any 3-cocycle cohomologous to $f$ may be constructed by changing the choices of $\alpha, v$ within the obvious limits.

Proof. This is identical with the proof given in [21, Chapter 4] for the purely algebraic case, and we do not repeat it here.

Q.E.D.

The unique element of $H_{\theta}^{3}(G ; \mathcal{U}(\mathscr{L}(\mathscr{M})))$ determined by $\{\mathscr{M}, \theta\}$ via Lemma 3.1 .5 will be denoted $f\{\mathscr{M}, \theta\}$, In the notations of 2.1.1 and the discussion preceding 3.1.5, the 3-cocycle $f$ satisfies $f=\partial v$.

Theorem 3.1.6. Let $\{\mathscr{M}, \theta\}$ be a G-kernel. Then $\{\mathscr{M}, \theta\}$ is extendable if and only if $f\{\mathscr{M}, \theta\}=$ identity in $H_{\theta}^{3}(G ; \mathcal{U}(\mathscr{Z}(\mathscr{M})))$.

Proof. (i) Let $\{\mathscr{M}, I, \pi\}$ be an extension of $\mathscr{M}$ by $G$ realizing $\theta$. We identify $\mathscr{M}$ with its image $I(\mathscr{M})$ in $\mathscr{N}$. By definition $g \rightarrow \alpha_{g}=\operatorname{Ad} \pi(g) \in \operatorname{Aut}(\mathscr{M})$ is a Borel lifting of $\theta$ into $\operatorname{Aut}(\mathscr{M})$. Since $\operatorname{Ad} \pi(g) \circ \operatorname{Ad} \pi(h)=\operatorname{Ad}\left(\pi(g) \pi(h) \pi(g h)^{*}\right) \circ$ $\operatorname{Ad} \pi(g h)$, and $(g, h) \rightarrow \pi(g) \pi(h) \pi(g h)^{*} \in \mathcal{U}(\mathscr{M})$ is Borel, we may choose $v(g, h)$ $=\pi(g) \pi(h) \pi(g h)^{*}$. But then

$$
\begin{aligned}
& \alpha_{g}(v(h, k)) v(g, h k) \\
= & \pi(g) \pi(h) \pi(k) \pi(h k)^{*} \pi(g)^{*} \pi(g) \pi(h k) \pi(g h k)^{*} \\
= & \pi(g) \pi(h) \pi(k) \pi(g h k)^{*} \\
= & \pi(g) \pi(h) \pi(g h)^{*} \pi(g h) \pi(k) \pi(g h k)^{*} \\
= & v(g, h) v(g h, k) \quad \text { for } g, h, k \in G .
\end{aligned}
$$

Thus for this choice of $\alpha, v, f(g, h, k)=1$, and $f\{\mathscr{M}, \theta\}$ is trivial.

(ii) Suppose $f\{\mathscr{M}, \theta\}=$ identity. By 3.1.5 (iii) we may choose Borel maps $\alpha: g \in G \rightarrow \alpha_{g} \in \operatorname{Aut}(\mathscr{M}), v:(g, h) \in G \times G \rightarrow v(g, h) \in \mathcal{U}(\mathscr{M})$ satisfying $\varepsilon \circ \alpha=\theta$, $\alpha_{g} \circ \alpha_{h}=\operatorname{Ad} v(g, h) \circ \alpha_{g h}$ and $v(g, h) v(g h, k)=\alpha_{g}(v(h, k)) v(g, h k)$ for all $g, h, k \in G$. Let $\mathscr{M}$ act on the Hilbert space $\mathscr{H}$, and define operators $I(x), x \in \mathscr{M}$, and $\pi(g)$, $g \in G$ on $L^{2}(G ; \mathcal{H})$ by

$$
\begin{array}{lll}
(I(x) \xi)(h)=\alpha_{h^{-1}}(x) \xi(h), & & \xi \in L^{2}(G ; \mathcal{H}), \\
(\pi(g) \xi)(h)=v\left(h^{-1}, g\right) \xi\left(g^{-1} h\right), & & \xi \in L^{2}(G ; \mathcal{H}) .
\end{array}
$$

We let $\Re$ be the von Neumann algebra on $L^{2}(G ; \mathscr{H})$ generated by the operators $I(x), x \in \mathscr{M}$, and $\pi(g), g \in G$. Clearly $I$ is an isomorphism of $\mathscr{M}$ into a subalgebra of $\Re$, and we may compute 


$$
\begin{aligned}
\left(\pi(g) I(x) \pi(g)^{*} \xi\right)(h) & =v\left(h^{-1}, g\right) \alpha_{h^{-1}}(x) v\left(h^{-1}, g\right)^{*} \xi(h) \\
& =\alpha_{h^{-1} \circ \alpha_{g}(x) \xi(h)} \\
& =\left(I\left(\alpha_{g}(x)\right) \xi\right)(h), \quad \text { for } g \in G, x \in \mathscr{M} .
\end{aligned}
$$

Thus $\pi(g) I(x) \pi(g)^{*}=I\left(\alpha_{g}(x)\right)$ and $\pi(g) I(\mathscr{M}) \pi(g)^{*}=I(\mathscr{M})$. Also

$$
\begin{aligned}
\left(\pi(g) \pi(h) \pi(g h)^{* \xi}\right)(k) & =v\left(k^{-1}, g\right) v\left(k^{-1} g, h\right) v\left(k^{-1}, g h\right)^{*} \xi(k) \\
& =\alpha_{k^{-1}}(v(g, h)) \xi(k) \\
& =(I(v(g, h)) \xi(k) \quad \text { for } g, h \in G .
\end{aligned}
$$

So $\pi(g) \pi(h)=I(v(g, h)) \pi(g h) \in I(\mathscr{M}) \pi(g h) . \quad\{\mathscr{l}, I, \pi\}$ is thus an extension of $\mathscr{M}$ by $G$, which, by the first computation above, realizes the kernel $\{\mathscr{M}, \theta\}$. Q.E.D.

The extension constructed above constitutes a generalization of the twisted crossed product studied in [27], [32]. As in [30], it is readily verified that the von Neumann algebra $\mathscr{I}$ does not depend on realization of $\mathscr{M}$ on the Hilbert space $\mathcal{H}$. Although there are many extensions realizing a given kernel (in general), we refer to any extension of $\mathscr{M}$ by $G$ constructed as in 3.1 .6 (ii) above as a regular extension of $\mathscr{M}$ by $G$.

In case the automorphisms $\alpha_{g}$ of 3.1 .6 (ii) are implemented by a Borel family $g \rightarrow u_{g}$ of unitaries on the Hilbert space $\mathcal{H}$ of $\mathscr{M}$, the generators $I(x)$, $\pi(g)$ of the regular extension may be represented as follows on $L^{2}(G ; \mathscr{H}) \simeq \mathscr{H} \otimes$ $\left.L^{2}(G)\right)$;

$$
\begin{aligned}
& I^{\prime}(x) \xi=(x \otimes 1) \xi, x \in \mathscr{M}, \xi \in L^{2}(G ; \mathcal{H}), \\
& \left(\pi^{\prime}(h) \xi\right)(g)=u_{g}^{*} *^{-1}\left(g^{-1}, h\right) u_{g^{-1}} \xi\left(h^{-1} g\right), h \in G .
\end{aligned}
$$

Indeed, these are the transforms of the generators $I(x), \pi(g)$ by the unitary operator $U$ on $L^{2}(G ; \mathcal{H})$ defined by $(U \xi)(g)=u_{g-1}^{*} \xi(g)$. For the purposes of the next result, it is more convenient to use these alternate generators.

Proposition 3.1.7. Let $1 \rightarrow M \rightarrow N \rightarrow G \rightarrow 1$ be any exact sequence of locally compact separble groups, and let $\mathscr{M}, \mathcal{I}$ be the von Neumann algebras generated by the left regular representations $\lambda^{M}, \lambda^{N}$ of $M, N$ respectively. Then $\Omega$ is a regular extension of $\mathscr{M}$ by $G$.

Proof. Let $g \in G \rightarrow n_{g} \in N$ be a Borel right inverse to the surjection $N \rightarrow G$; we regard $M$ as being a normal subgroup of $G$. As is well known (see e.g. [20]), $N$ may be identified (as a standard Borel group) with $M \times G$ endowed with the multiplication $(m, g)\left(m^{\prime}, g^{\prime}\right)=\left(m \beta_{g}\left(m^{\prime}\right) m\left(g, g^{\prime}\right), g g^{\prime}\right)$ where $\beta_{g}(m)=$ $n_{g} m n_{g}^{-1}$ and $m(g, h)=n_{g} n_{h} n_{g h}^{-1} \in M$; a right Haar measure on $N$ is given by 
the product of right Haar measures on $M$ and $G$.

Let $\alpha_{g} \in \operatorname{Aut}(M)$ be defined by $\alpha_{g}(m)=n_{g^{-1}} 1 m n_{g^{-1}}$, and set $v(g, h)$ $=n_{g}^{-1} 1 n_{h}^{-1}-1 n_{h^{-1}} g^{-1} \in M$; we also denote by $v(g, h)$ the unitary $\lambda^{M}(v(g, h)) \in \mathscr{M}$. Each automorphism $\alpha_{g}$ defines an automorphism, also denoted $\alpha_{g}$, of $\mathscr{M}$, which is implemented by the unitary $u_{g},\left(u_{g} \xi\right)(m)=\delta(g)^{-1 / 2} \xi\left(\alpha_{g}^{-1}(m)\right), g \in G, m \in$ $M, \xi \in L^{2}(M)$, where $\delta(g)$ is the module of $\alpha_{g}$. Under the canonical identifications of $L^{2}\left(G ; L^{2}(M)\right), L^{2}(M \times G)$ and $L^{2}(N)$, it is a routine matter to verify that the generators $I^{\prime}\left(\lambda^{M}(m)\right), \pi^{\prime}(g)$ for $m \in M, g \in G$, of the regular extension of $\mathscr{M}$ by $G$ determined by the choice of $\alpha, v$ above, correspond to $\lambda^{N}(m), m \in G$ and $\lambda^{N}\left(n_{g}^{-1}-1\right), g \in G$, respectively. We leave the details to the reader. Q.E.D.

Much of the value of crossed products stems from the Duality Theorem of [30], in case the group $G$ is abelian. While it seems to be very difficult, and perhaps impossible, to phrase a duality theory for twisted crossed products or regular extensions by abelian groups, some aspects of the theory persist ${ }^{1)}$. For the balance of this section, the group $G$ will be supposed to be abelian, with dual group $\hat{G}$.

Following [30], define for $p \in \hat{G}$ a unitary $\mu(p)$ on $L^{2}(G ; \mathcal{H})$ by $(\mu(p) \xi)(g)$ $=\overline{\langle g, p\rangle} \xi(g)$. Let $\{\mathscr{N}, I, \pi\}$ be a regular extension of $\mathscr{M}$ by $G$, where $\mathscr{M}$ acts on $\mathcal{H}$. Evidently we have

$$
\begin{aligned}
& \mu(p) I(x) \mu(p)^{*}=I(x), x \in \mathscr{M}, \\
& \mu(p) \pi(g) \mu(p)^{*}=\overline{\langle g, p\rangle} \pi(g), g \in G .
\end{aligned}
$$

Thus $p \rightarrow \hat{\alpha}_{p}=\operatorname{Ad} \mu(p)$ defines a continuous representation of $\hat{G}$ in $\operatorname{Aut}(\mathscr{R})$, called the dual action.

Proposition 3.1.8. The fixed point algebra of a regular extension $\{\eta$, $I, \pi\}$ of $\mathcal{M}$ by an abeliang roap $G$ under the dual action is precisely $I(\mathscr{M})$.

Proof. We may suppose that $\mathscr{M}$ is in standard form on $\mathscr{H}$, and that $I(x)$, $x \in \mathscr{M}, \pi(g), g \in G$ are given on $L^{2}(G ; \mathscr{H})$ by

$$
(I(x) \xi)(g)=\alpha_{g^{-1}}(x) \xi(g),(\pi(h) \xi)(g)=v\left(g^{-1}, h\right) \xi\left(h^{-1} g\right) .
$$

From [14], we may choose unitaries $W^{g}, g \in G$ on $\mathcal{H}$ such that $\operatorname{Ad} W^{g}=\alpha_{g^{-1}}$ on $\mathcal{M}$. Thus

$$
\operatorname{Ad} W^{g} W^{h}=\alpha_{g^{-10}} \alpha_{h^{-1}}
$$

1) An adequate duality theory, submitted to the Pacific Journal, has been developed by Y. Nakagami and the author. 


$$
=\operatorname{Ad} v\left(g^{-1}, h^{-1}\right) W^{g h}, \text { as } G \text { is abelian. }
$$

Thus $W^{g} W^{h}=c(g, h) v\left(g^{-1}, h^{-1}\right) W^{g h}$, where $(g, h) \rightarrow c(g, h) \in \mathcal{U}\left(\mathscr{M}^{\prime}\right)$ is Borel.

Define, for $h \in G$, a unitary $\rho(h)$ on $L^{2}(G ; \mathcal{H})$ by

$$
(\rho(h) \xi)(g)=\delta_{G}(h)^{-1 / 2} W^{g}\left(W^{g h}\right)^{*} c(h, g)^{*} \xi(g h),
$$

where $\delta_{G}$ is the modular function of $G$. We claim that $\{\rho(g), g \in G\}$ commutes with $\Re$. First, compute

$$
\begin{aligned}
& \left(\rho(h) I(x) \rho(h)^{*} \xi\right)(g) \\
= & W^{g}\left(W^{g h}\right)^{*} c(h, g)^{*} \alpha_{(g h)^{-1}}(x) c(h, g)\left(W^{g h}\right)\left(W^{g}\right)^{*} \xi(g) \\
= & \alpha_{g^{-1}} \alpha_{(g h)^{-1}}^{-1} \alpha_{(g h)^{-1}}(x) \xi(g) \\
= & \alpha_{g^{-1}(x) \xi(g)} \\
= & (I(x) \xi)(g), \quad \text { for } \quad x \in \mathscr{M}, \xi \in L^{2}(G ; \mathcal{H}) .
\end{aligned}
$$

Secondly, we have for $h, k \in G, \xi \in L^{2}(G ; \mathcal{H})$

$$
(\rho(h) \pi(k) \xi)(g)=\delta_{g}(h)^{-1 / 2} W^{g}\left(W^{g h}\right)^{*} c(h, g)^{*} v\left(h^{-1} g^{-1}, k\right) \xi\left(k^{-1} g h\right)
$$

and

$$
\begin{aligned}
(\pi(k) \rho(h) \xi)(g) & =v\left(g^{-1}, k\right)(\rho(h) \xi)\left(k^{-1} g\right) \\
& =v\left(g^{-1}, k\right) \delta_{G}(h)^{-1 / 2} W^{k^{-1} g}\left(W^{k^{-1} g h}\right)^{*} c\left(h, k^{-1} g\right)^{*} \xi\left(k^{-1} g h\right) .
\end{aligned}
$$

On the other hand, using the relationship between $W^{g}, c(g, h), v(g, h)$ given above, and the fact that $\partial v=1$, we see

$$
\begin{aligned}
& v\left(g^{-1}, k\right) W^{k^{-1}} g\left(W^{k^{-1} g h}\right)^{*} c\left(h, k^{-1} g\right)^{*} \\
= & v\left(g^{-1}, k\right)\left(W^{h}\right)^{*} c\left(h, k^{-1} g\right) v\left(h^{-1}, g^{-1} k\right) c\left(h, k^{-1} g\right)^{*} \\
= & \left(W^{h}\right)^{*} \alpha_{k^{-1}}\left(v\left(g^{-1}, k\right)\right) v\left(h^{-1}, g^{-1} k\right) \\
= & \left(W^{h}\right)^{*} v\left(h^{-1}, g^{-1}\right) v\left(h^{-1} g^{-1}, k\right) .
\end{aligned}
$$

Furthermore

$$
\begin{aligned}
& W^{g}\left(W^{g h}\right)^{*} c(h, g)^{*} \\
= & \left(W^{h}\right)^{*} c(h, g) v\left(h^{-1}, g^{-1}\right) c(h, g)^{*} \\
= & \left(W^{h}\right)^{*} v\left(h^{-1}, g^{-1}\right)
\end{aligned}
$$

Thus $\rho(h) \pi(k)=\pi(k) \rho(h)$ for all $h, k \in G$ as required, and $\{\rho(h) ; h \in G\} \subseteq \mathscr{I}^{\prime}$. It is clear that for $y \in \mathscr{H}^{\prime}$, the operator $\bar{y}=y \otimes 1$ on $L^{2}(G ; \mathscr{H})$ is also in $\mathscr{I}^{\prime}$; thus $\mathscr{I} \subseteq\left(\mathscr{M}^{\prime} \otimes 1\right)^{\prime}=\mathscr{M} \otimes \mathscr{B}\left(L^{2}(G)\right)$. On the other hand, as $\{\mu(p), p \in \hat{G}\}$ generates the von Neumann algebra $1 \otimes L^{\infty}(G)$ on $L^{2}(G ; \mathcal{H})$, any fixed point in $\mathscr{B}(\mathcal{K}) \otimes \mathscr{B}\left(L^{2}(G)\right)$ of $\left\{\alpha_{p}: p \in G\right\}$ lies in $\mathscr{B}(\mathcal{H}) \otimes L^{\infty}(G)$. Thus, the fixed points 
in $\mathscr{N}$ lie in $\left(\mathscr{M} \otimes \mathscr{B}\left(L^{2}(G)\right) \cap\left(\mathscr{B}(\mathscr{H}) \otimes L^{\infty}(G)\right)=\mathscr{M} \otimes L^{\infty}(G)\right.$.

It is thus sufficient to show $\mathscr{N} \cap\left(\mathscr{M} \otimes L^{\infty}(G)\right)=I(\mathscr{M})$. Let $x$ lie in the indicated intersection, so that the action of $x$ on $\xi \in L^{2}(G ; \mathcal{H})$ may be represented by $(x \xi)(g)=x(g) \xi(g)$ for some bounded Borel map $x: G \rightarrow \mathscr{M}$. On the other hand, $x$ commutes with $\rho(h), h \in G$, and

$$
(\rho(h) x \xi)(g)=\delta_{G}(h)^{-1 / 2} W^{g}\left(W^{g h}\right)^{*} c(h, g)^{*} x(g h) \xi(g h)
$$

while

$$
(x \rho(h) \xi)(g)=\delta_{G}(h)^{-1 / 2} x(g) W^{g}\left(W^{g h}\right) c(h, g)^{*} \xi(g h) .
$$

Thus we require that for each $h \in G, W^{g}\left(W^{g h}\right)^{*} x(g h)=x(g) W^{g}\left(W^{g h}\right)^{*}$, or $\alpha_{g^{-1}}$ 。 $\alpha_{h^{-1} g^{-1}}^{-1}(x(g h))=x(g)$, for almost all $g \in G$. Thus $\alpha_{g^{-1}}^{-1}(x(g))$ is almost everywhere independent of $g$; we write $x_{0}$ for this fixed value. It is clear that $x$ and $I\left(x_{0}\right)$ represent the same element of $\mathscr{M} \otimes L^{\infty}(G)$, so that $x \in I(\mathscr{M})$. The converse inclusion, $I(\mathscr{M}) \subset \mathscr{R} \cap\left(\mathscr{M} \otimes L^{\infty}(G)\right)$ is automatic. $\quad$ Q.E.D.

Remark. It is virtually certain that the operators $\rho(h), h \in G$, and $\bar{y}=$ $y \otimes 1, y \in \mathscr{M}$, defined in the proof of 3.1.8, generate the commutant of $\mathscr{N}$. The author however has not checked this in full detail. In Section 5, we shall establish a kind of converse to 3.1.8.

\subsection{Operations on Kernels}

In 3.1.5 we have associated to each $G$-kernel $\{\mathscr{M}, \theta\}$ an element $f\{\mathscr{M}, \theta\}$ $\in H_{\theta}^{3}(G ; \mathcal{U}(\mathscr{Z}(\mathscr{M})))$. Here we seek to answer two natural questions which arise; which 3-cocycles arise in this manner; and what are the operations on kernels corresponding to the natural group structure in $H_{\theta}^{3}$ ?

Finding the "inverse" of a kernel is the simplest of these questions and we adress this first.

Let $\mathscr{M}$ be a von Neumann algebra, and $\mathscr{M}^{\circ}$ the opposite algebra i.e. $\mathscr{M}^{\circ}$ has the same ring and involutive structure as $\mathscr{M}$, but the product is $i(x) i(y)$ $=i(y x)$ (where, for $x \in \mathscr{M}, i(x)$ denotes the corresponding element of $\mathscr{M}^{\circ}$ ). For $\alpha \in \operatorname{Aut}(\mathscr{M})$, set $\alpha^{0}(i(x))=i(\alpha(x))$; it is readily checked that $\alpha \rightarrow \alpha^{0}$ is an isomorphism (and homeomorphism) of $\operatorname{Aut}(\mathscr{M})$ with $\operatorname{Aut}\left(\mathscr{M}^{\circ}\right)$, and that $(\operatorname{Ad} u)^{\circ}$ $=\operatorname{Ad} i(u) *$ for $u \in \mathcal{U}(\mathscr{M})$. Thus if $\{\mathscr{M}, \theta\}$ is a $G$-kernel, and $\alpha: G \rightarrow \operatorname{Aut}(\mathscr{M})$ a Borel map with $\varepsilon \circ \alpha=\theta$, then we may define a new $G$-kernel $\left\{\mathscr{H}^{\circ}, \theta^{\circ}\right\}$ by $\theta_{g}^{o}=$ $\varepsilon\left(\alpha_{g}^{o}\right)$; trivially, $\theta^{\circ}$ depends only on $\theta$. Note also that if $\alpha_{g} \circ \alpha_{h}=\operatorname{Ad} v(g, h) \circ \alpha_{g h}$, then $\alpha_{g}^{o} \circ \alpha_{h}^{o}=\mathrm{Ad} v^{o}(g, h) \circ \alpha_{g h}^{o}$, where $v^{o}(g, h)=i\left(v(g, h)^{*}\right) \in \mathcal{U}\left(\mathscr{M}^{o}\right)$. Finally, if $\alpha_{g}(v(h, k)) v(g, h k)=f(g, h, k) v(g, h) v(g h, k)$, then $\alpha_{g}^{o}\left(v^{o}(g, k)\right) v^{o}(g, h k)=i\left(f(g, h, k)^{*}\right)$ 
$\times v^{0}(g, h) v^{\circ}(g h, k)$. However, the map $i: \mathscr{Z}(\mathscr{M}) \rightarrow \mathscr{Z}\left(\mathscr{M}^{\circ}\right)$ is an isomorphism which intertwines the natural actions $\theta, \theta^{\circ}$ of $G$ on $\mathscr{Z}(\mathscr{M}), \mathscr{Z}\left(\mathscr{H}^{\circ}\right)$ respectively. Thus we have shown

Proposition 3.2.1. Let $\{\mathscr{M}, \theta\}$ be a G-kernel, and $\left\{\mathscr{M}^{\circ}, \theta^{\circ}\right\}$ the opposite kernel as above. Then $\left.f\left\{\mathscr{M}^{0}, \theta^{\circ}\right\}=f_{\{\mathfrak{H}}^{-1} \mathscr{\mathscr { N }}, \theta\right\}$ in $H_{\theta}^{3}(G ; \mathcal{U}(\mathscr{Z}(\mathscr{M})))$.

The product structure among kernels is somewhat more complex, and seems to depend on the following idea. Essentially, we need to be able to form the "tensor product of von Neumann algebras over their common centre". Specifically, let $\mathscr{M}, \overline{\mathscr{M}}$ be von Neumann algebras with centres $\mathscr{Z}, \bar{Z}$ and let $\Phi: \mathscr{Z} \rightarrow \bar{Z}$ be a fixed isomorphism. Let $\mathscr{M}=\int_{\Gamma}^{\oplus} \mathscr{M}(r) d \mu(\gamma)$ and $\overline{\mathscr{M}}=\int_{\Gamma}^{\oplus} \overline{\mathscr{M}}(r) d \mu(\gamma)$ be the central decompositions of $\mathscr{M}$ and $\mathscr{M}$, where the index spaces for the decompositions are identified by means of the isomorphism $\Phi$.

Definition 3.2.2. $\mathscr{M} \otimes_{\Phi} \overline{\mathscr{M}}$ is the von Neumann algebra whose central decomposition is $\int_{\Gamma}^{\oplus} \mathscr{M}(r) \otimes \overline{\mathscr{M}}(r) d \mu(\gamma)$.

The dependence of $\mathscr{M} \otimes_{\Phi} \overline{\mathscr{M}}$ on $\Phi$ is strong; if $\mathscr{Z}$ is two-dimensional and $\mathscr{M}=\mathscr{M}_{1} \oplus \mathscr{M}_{2}, \overline{\mathscr{M}}=\overline{\mathscr{M}}_{1} \oplus \overline{\mathscr{M}}_{2}$, then $\mathscr{M} \otimes_{\Phi} \overline{\mathscr{M}}$ can be either of $\mathscr{M}_{1} \otimes \overline{\mathscr{M}}_{1} \oplus \mathscr{M}_{2}$ $\otimes \overline{\mathscr{M}}_{2}$ or $\mathscr{M}_{1} \otimes \overline{\mathscr{M}}_{2} \oplus \mathscr{M}_{2} \otimes \overline{\mathscr{M}}_{1}$, and these may well be non-isomorphic.

Let $\{\mathscr{M}, \theta\},\{\overline{\mathscr{M}}, \bar{\theta}\}$ be $G$-kernels, and choose Borel maps $\alpha$ (resp. $\bar{\alpha}$ ): $G \rightarrow \operatorname{Aut}(\mathscr{M})(\operatorname{resp} . \operatorname{Aut}(\overline{\mathscr{M}})), v($ resp. $\bar{v}): G \times G \rightarrow \mathcal{U}(\mathscr{M})($ resp. $\mathcal{U}(\overline{\mathscr{M}})$ ) with

$$
\begin{aligned}
& \alpha_{g} \circ \alpha_{h}=\operatorname{Ad} v(g, h) \circ \alpha_{g h} \quad \bar{\alpha}_{g} \circ \bar{\alpha}_{h}=\operatorname{Ad} \bar{v}(g, h) \circ \bar{\alpha}_{g h} \\
& \alpha_{g}(v(h, k)) v(g, h k)=f(g, h, k) v(g, h) v(g h, k) \\
& \bar{\alpha}_{g}(\bar{v}(h, k)) \bar{v}(g, h k)=\bar{f}(g, h, k) \bar{v}(g, h) \bar{v}(g h, k), \quad g, h, k \in G .
\end{aligned}
$$

In order to consider the product $f \bar{f}$ we must assume there is an isomorphism $\Phi: \mathscr{Z}(\mathscr{M}) \rightarrow \mathscr{Z}(\bar{M})$ with $\bar{\theta}_{g} \circ \Phi=\Phi \circ \theta_{g}, g \in G$; we shall consider $\mathscr{Z}(\mathscr{M})$ and $\mathscr{Z}(\overline{\mathscr{M}})$ to be identified by means of $\Phi$. We choose once and for all point realization $(g, \gamma) \rightarrow g \gamma$ for the action of $G$ on $L^{\infty}(\Gamma, \mu) \simeq \mathscr{Z}(\mathscr{M}) \simeq \mathscr{Z}(\overline{\mathscr{M}})$ corresponding to $\theta$ and $\bar{\theta}$. If $G$ is discrete, we may, by 2.2 .2 , assume that there are fields of isomorphisms $\kappa_{(g, \gamma)}$ (resp. $\left.\bar{\kappa}_{(g, \gamma)}\right): \mathscr{M}(\gamma) \rightarrow \mathscr{H}(g \gamma)$ (resp. $\overline{\mathscr{M}}(r) \rightarrow$ $\overline{\mathscr{M}}(g r))$ with $\kappa_{(g, \gamma)}(\bar{x}(\gamma))=\left(\alpha_{g}(x)\right)\left(g_{\gamma}\right)$ (resp. $\left.\bar{\kappa}_{(g, \gamma)}(\bar{x}(r))=\left(\alpha_{g}(\bar{x})\right)(g r)\right)$, where $\mathscr{M}=\int_{\Gamma}^{\oplus} \mathscr{M}(r) d \mu(\gamma)\left(\right.$ resp. $\left.\overline{\mathscr{M}}=\int_{\Gamma}^{\oplus} \overline{\mathscr{M}}(\gamma) d \mu(\gamma)\right)$ is the central decomposition and $x \sim x(r)$ (resp. $\bar{x} \sim \bar{x}(\gamma)$ ).

Proposition 3.2.3. With notation as above, let $\mathscr{N}=\mathscr{M} \otimes_{\Phi} \overline{\mathscr{M}}$, and define 
automorphisms $\beta_{g}, g \in G$ on $\mathscr{N}$ by

$$
\left(\beta_{g}(y)\right)(g \gamma)=\left(\kappa_{(g, \gamma)} \otimes \kappa_{(g, \gamma)}\right)(y(\gamma)), y \in \mathscr{l}, y \sim y(\gamma) .
$$

Then (i) $\beta_{g} \beta_{h} \beta_{g h}^{-1} \in \operatorname{Int}(\mathscr{N})$

(ii) if $\varphi_{g}=\varepsilon\left(\beta_{g}\right)$, then $f\{\Re, \varphi\}=f\{\mathscr{M}, \theta\} f\{\bar{M}, \bar{\theta}\}$.

Proof. The fact that the $\beta_{g}$ are indeed automorphisms of follows trivially from the properties of the isomorphisms $\kappa_{(g, \gamma)}, \bar{\kappa}_{(g, \gamma)}$. For (i), we compute, for $y \sim y(r)$

$$
\left(\beta_{g} \beta_{h} \beta_{g h}^{-1}(g)\right)(\gamma)=\left(\nu_{(g, h, \gamma)} \otimes \bar{v}_{(g, h, \gamma)}\right)(y(\gamma)),
$$

where

$$
\nu_{(g, h, \gamma)}=\kappa_{\left(g, g^{-1} \gamma\right)} \circ \kappa_{\left.\left(h, h^{-1} g^{-1} \gamma\right) \circ \kappa_{\left(g h, h^{-1}\right.}^{-1} g^{-1} \gamma\right)}
$$

and similarly for $\bar{v}$. Since $\alpha_{g} \circ \alpha_{h} \circ \alpha_{g h}^{-1}=\operatorname{Ad} v(g, h)$ we see $\nu_{(g, h, \gamma)}=\operatorname{Ad} v_{\gamma}(g, h)$ $\mu$ a.e., where $v(g, h)=\int_{\Gamma}^{\oplus} v_{\gamma}(g, h) d \mu(\gamma)$ is the central decomposition. A similar statement holds for $\bar{v}_{(g, h, \gamma)}$, so that $\beta_{g} \beta_{h} \beta_{g h}^{-1}=\operatorname{Ad} u(g, h)$, where $u(g, h) \in \mathcal{N}$ has central decomposition $u(g, h)=\int_{\Gamma}^{\oplus} v_{\gamma}(g, h) \otimes \bar{v}_{\gamma}(g, h) d \mu(\gamma)$.

Finally, since both $f(g, h, k)$ and $\bar{f}(g, h, k)$ become diagonal operators in the central decompositions of $\mathscr{M}$ and $\bar{M}$, we see

$$
\beta_{g}(u(h, k)) u(g, h k)=f(g, h, k) \bar{f}(g, h, k) u(g, h) u(g h, k)
$$

for $g, h, k \in G$, and (ii) is established.

Q.E.D.

In order to see that all 3-cocycles arise as obstructions it seems to be necessary to restrict attention to countable groups $G$. The construction below is somewhat related to that of 2.1.4.

Proposition 3.2.4. Let $G$ be a countable group with order $\neq 2, \theta$ a homomorphism of $G$ into $\operatorname{Aut}(\mathcal{A})$, where $\mathcal{A}$ is an abelian von Neumann algebra, and $\tilde{f} \in H_{\theta}^{3}(G ; \mathcal{U}(\mathcal{A}))$. Then there is a G-kernel $\{\mathscr{M}, \theta\}$ with $f\{\mathscr{M}, \theta\}=\tilde{f}$.

Proof. Let $f \in \tilde{f}$ be a normalized 3-cocycle, so that

$$
\theta_{g}(f(h, k, l)) f(g, h k, l) f(g, h, k)=f(g h, k, l) f(g, h, k l)
$$

We shall construct a full $\mathrm{II}_{1}$-factor $\mathscr{P}$, automorphisms $\alpha_{g}, g \in G$ of $\mathscr{M}=\mathscr{A} \otimes \mathscr{Q}$ with $\alpha_{g}=\theta_{g} \otimes \imath$ on $\mathcal{A} \otimes 1$, and unitaries $v(g, h) \in \mathscr{M}$ with $\alpha_{g} \circ \alpha_{h}=\operatorname{Ad} v(g, h) \circ \alpha_{g h}$ and $\alpha_{g}(v(h, k)) v(g, h k)=f(g, h, k) v(g, h) v(g h, k)$ for $g, h, k \in G$.

Choose once and for all a point realization $(g, \gamma) \in G \times \Gamma \rightarrow g \gamma \in \Gamma$ of 
the given action $\theta$ of $G$ on $\mathcal{A}$. Let $\Phi_{G}$ denote the free group on the generators $\{[g, h], g \neq e \neq h$ in $G\}$ (each $[g, h]$ is a single symbol, not a commutator), and let $\mathcal{Q}$ denote the von Neumann algebra generated by the left regular representation $\lambda$ of $\Phi_{G}$. Since each of central unitaries $f(g, h, k)$ may be represented by a Borel function $\gamma \in \Gamma \rightarrow f_{\gamma}(g, h, k)$ with $\left|f_{\gamma}(g, h, k)\right|=1$, we may for each $\gamma \in \Gamma$ define automorphisms $\beta_{g}^{\gamma}$ of $\mathcal{Q}$ by

$$
\beta_{g}^{\gamma}\left(\lambda([h, k])=f_{\gamma}(g, h, k) \lambda([h, k]), g, h, k \in G\right.
$$

(see [23]).

We view $A \otimes \mathscr{Q}$ as the direct integral of the constant field $r \rightarrow \mathscr{L}(r)=\mathscr{Q}$, $r \in \Gamma$, of von Neumann algebras; with $\beta_{g}=\int_{\Gamma}^{\oplus} \beta_{g}^{\gamma} d \mu(\gamma), \beta_{g} \in \operatorname{Aut}(\mathcal{A} \otimes \mathscr{P})$ and we have $\beta_{g}(a \otimes 1)=a \otimes 1$, and $\beta_{g}(1 \otimes \lambda[h, k])=f(g, h, k) \otimes \lambda[h, k]$. From the arguments of $\left[21\right.$; page 130] the maps $[h, k] \rightarrow[g, h][g h, k][g, h k]^{-1}$ from the generators of $\Phi_{G}$ into $\Phi_{G}$ extend to automorphisms of $\Phi_{G}$. By familiar arguments, there are thus automorphisms $\delta_{g}, g \in G$ of $\mathscr{P}$ with $\delta_{g}(\lambda([h, k]))=$ $\lambda\left([g, h][g h, k][g, h k]^{-1}\right)$.

We may now define the desired automorphisms of $\mathscr{A} \otimes \mathscr{Q}$ by

$$
\alpha_{g}=\left(\iota \otimes \delta_{g}\right) \circ \beta_{g} \circ\left(\theta_{g} \otimes \iota\right) . \quad \text { Clearly } \alpha_{g}(a \otimes 1)=\theta_{g}(a) \otimes 1
$$

for $a \in \mathcal{A}$, while, writing $v(h, k)=1 \otimes \lambda([h, k])$, we have

$$
\alpha_{g}(v(h, k))=(f(g, h, k) \otimes 1) v(g, h) v(g h, k) v(g, h k)^{*} .
$$

Also $\alpha_{g} \circ \alpha_{h}(a \otimes 1)=\alpha_{g h}(a \otimes 1)$ for $a \in \mathcal{A}$; on the other hand an easy computation using the cocycle identity for $f$ (c.f. [21]) shows that for $g, h, k, l \in G$, $\alpha_{g} \circ \alpha_{h}(v(k, l))=v(g, h) \alpha_{g h}(v(k, l)) v(g, h)^{*}$ so that $\alpha_{g} \circ \alpha_{h}=\operatorname{Ad} v(g, h) \circ \alpha_{g h}$ as required.

Q.E.D.

Remarks. (i) In case $G=Z_{p}$, the $p$-element cyclic group, and $\mathcal{A}=\mathbb{C}$, Connes [5] has constructed automorphisms of the hyper-finite $\mathrm{II}_{1}$-factor with specified arbitrary obstruction. It is not clear to what extent this construction may be generalized ${ }^{1)}$.

(ii) The above proposition remains true in the exceptional case $G=\mathbb{Z}_{2}$. However it has somewhat limited interest as if $\mathbb{Z}_{2}$ acts freely on the abelian von Neumann algebra $\mathcal{A}, H^{3}(G ; \mathcal{U}(\mathcal{A}))=\{0\}$.

1) V. Jones, in a preprint entitled "Actions of finite abelian groups on the hyperfinite $\mathrm{II}_{1}$ factor" has realized an arbitrary obstruction for a finite abelian group in the hyperfinite $\mathrm{II}_{1}$ factor. 


\section{§4. Split Kernels and Their Extensions}

\subsection{Splitting Criteria}

Definition 4.1.1. A $G$-kernel $\{\mathscr{M}, \theta\}$ is said to be split in case there is a Borel homomorphism $\alpha: G \rightarrow \operatorname{Aut}(\mathscr{H})$ with $\varepsilon \circ \alpha=\theta$. Such a map $\alpha$ will be termed a splitting map for $\theta$ or $\{\mathscr{M}, \theta\}$.

It is clear that the 3-cocycle associated to a split $G$-kernel is trivial; we shall establish a partial converse to this.

The type I case is easily disposed of by

Remark 4.12. If $\mathscr{M}$ is of type $\mathrm{I}$ it is of the form $\sum \oplus \mathcal{A}_{n} \otimes \mathscr{F}_{n}$ where $\mathcal{A}_{n}$ is abelian, and $\mathscr{F}_{n}$ is the type $I_{n}$-factor; thus it suffices to consider the case $\mathscr{M}=\mathscr{A}_{n} \otimes \mathscr{I}_{n}$. But then if $\alpha \in \operatorname{Aut}(\mathscr{M})$ and $\beta$ is the restriction of $\alpha$ to $\mathscr{A}_{n} \otimes 1$, then $\operatorname{Ad} u \circ \alpha=\beta$ for some $u \in \mathcal{U}(\mathscr{M})$. Thus if $g \rightarrow \alpha_{g} \in \operatorname{Aut}(\mathscr{M})$ is a Borel map with $g \rightarrow \beta_{g}$ a homomorphism ( $\beta_{g}$ is the restriction of $\alpha_{g}$ to $\mathcal{A}_{n} \otimes 1$ ), then $\operatorname{Ad} u_{g} \circ \alpha_{g}$ $=\beta_{g}$ for some map $g \rightarrow u_{g} \in \mathcal{U}(\mathscr{M})$ which we easily see may be chosen to be Borel. Thus $\varepsilon \circ \alpha=\theta$ is split.

Theorem 4.1.3. Let $\{\mathscr{M}, \theta\}$ be a G-kernel with trivial obstruction. Then if either $\mathscr{M}$ is properly infinite, or $\mathscr{M}$ is a finite $\mathrm{I}_{1}$-algebra and $G$ is finite, $\{\mathscr{M}, \theta\}$ is split.

Proof. Let $\{\mathscr{M}, \theta\}$ have trivial obstruction. By virtue of 3.1 .5 (iii) we may choose Borel maps $\alpha: G \rightarrow \operatorname{Aut}(\mathscr{M}), u: G \times G \mathcal{U}(\mathscr{M})$ satisfying

a) $\varepsilon \circ \alpha=\theta$,

b) $\alpha_{g} \circ \alpha_{h}=\operatorname{Ad} u(g, h) \circ \alpha_{g h} ; g, h \in G$,

c) $\alpha_{g}(u(h, k)) u(g, h k)=u(g, h) u(g h, k) ; \quad g, h, k \in G$.

These maps will be fixed throughout the remainder of the proof. We consider each case separately.

\section{Case 1: $\mathscr{M}$ properly infinite.}

Let $M_{\infty}(\boldsymbol{C})$ denote the $I_{\infty}$ factor. From the proof of Lemma 4.7 of [30], we see we may choose an isomorphism $\sigma: \mathscr{M} \rightarrow \mathscr{M} \otimes M_{\infty}(\boldsymbol{C})$, and unitaries $w_{g}$, $g \in G$ in $\mathscr{H} \otimes M_{\infty}(\boldsymbol{C})$ with $\mathrm{Ad} w_{g} \circ \sigma \circ \alpha_{g} \circ \sigma^{-1}=\alpha_{g} \otimes \iota$ (The only point in the proof of Takesaki's Lemma 4.7 where it is necessary to assume $g \rightarrow \alpha_{g}$ is a representation is in proving $w_{g} \gamma_{g}\left(w_{h}\right)=w_{g h}$ where $\gamma_{g}=\sigma \circ \alpha_{g} \circ \sigma^{-1}$.) By construction, $g \rightarrow w_{g}$ is Borel. 
By 2.1.3, there is a Borel map $g \in G \rightarrow v(g) \in \mathcal{U}\left(\mathscr{H} \otimes M_{\infty}(\boldsymbol{C})\right)$ with $u(g, h) \otimes 1$ $=\left(\alpha_{g} \otimes \iota\right)(v(h)) v(g) v(g h)^{*} ; g, h \in G$. Now

$$
\begin{aligned}
& \operatorname{Ad} v(g)^{*} \circ\left(\alpha_{g} \otimes \iota\right) \circ \operatorname{Ad} v(h)^{*} \circ\left(\alpha_{h} \otimes \iota\right) \\
= & \operatorname{Ad} v(g)^{*}\left(\alpha_{g} \otimes \iota\right)\left(v(h)^{*}\right) \circ\left(\alpha_{g} \circ \alpha_{h} \otimes \iota\right) \\
= & \operatorname{Ad} v(g)^{*}\left(\alpha_{g} \otimes \iota\right)\left(v(h)^{*}\right)(u(g, h) \otimes 1) \circ\left(\alpha_{g h} \otimes \iota\right) \\
= & \operatorname{Ad} v(g h)^{*} \circ\left(\alpha_{g h} \otimes \iota\right) .
\end{aligned}
$$

Thus $g \rightarrow \operatorname{Adv}(g)^{*} \circ\left(\alpha_{g} \otimes \iota\right)$ is a (Borel) representation of $G$ in $\operatorname{Aut}\left(\mathscr{M} \otimes M_{\infty}(\boldsymbol{C})\right)$. However

$$
\begin{aligned}
\operatorname{Ad} v(g)^{*} \circ\left(\alpha_{g} \otimes \imath\right) & =\operatorname{Ad} v(g)^{*} w_{g} \circ \sigma \circ \alpha_{g} \circ \sigma^{-1} \\
& =\sigma \circ\left(\operatorname{Ad} \sigma^{-1}\left(v(g)^{*} w_{g}\right) \circ \alpha_{g}\right) \circ \sigma^{-1}
\end{aligned}
$$

so that $g \rightarrow \operatorname{Ad} \sigma^{-1}\left(v(g)^{*} w_{g}\right) \circ \alpha_{g}=\beta_{g}$ is a representation of $G$ in $\operatorname{Aut}(\mathscr{M})$. Since $\varepsilon \circ \beta=\varepsilon \circ \alpha=\theta, \beta$ is a splitting map for $\theta$.

Case 2: $\mathscr{M}$ a $\mathrm{II}_{1}$-algebra, and $G$ finite of order $n$.

Let $\left\{e_{i j} ; i, j=1, \cdots, n\right\}$ be a system of $n \times n$ matrix units in $\mathscr{M}$, and $\mathscr{N}$ $=\mathscr{M} \cap\left\{e_{i j} ; i, j=1,2, \cdots, n\right\}^{\prime}$. Thus $\mathscr{M}$ is isomorphic with $\mathscr{N} \otimes M_{n}(\boldsymbol{C})$, and $\left\{e_{i j}\right\}$ correspond to the canonical matrix units $\left\{f_{i j} ; i, j=1, \cdots, n\right\}$ in the full $n \times n$ matrix algebra $M_{n}(\boldsymbol{C})$. We regard $\alpha_{g}, g \in G$ as automorphisms on

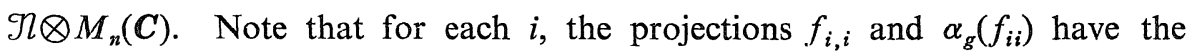
same centre-valued trace $\frac{1}{n}$, and hence are equivalent. We may thus find unitaries $w_{g}, g \in G$ in $\Re \otimes M_{n}(\boldsymbol{C})$ with $\operatorname{Ad} w_{g} \circ \alpha_{g}\left(f_{i j}\right)=f_{i j}$ for all $g$, and $i, j=$ $1,2, \cdots, n$. Thus we may write $\operatorname{Ad} w_{g} \circ \alpha_{g}=\beta_{g} \otimes \iota$ for some automorphisms $\beta_{g}$ of $\eta$.

Note that $\left(\beta_{g} \otimes \iota\right) \circ\left(\beta_{h} \otimes \imath\right)=\operatorname{Ad} w_{g} \alpha_{g}\left(w_{h}\right) u(g, h) w_{g h}^{*} \circ\left(\beta_{g h} \otimes \iota\right)$; thus $w_{g} \alpha_{g}\left(w_{h}\right)$ $\times u(g, h) w_{g h}^{*}=u_{1}(g, h) \otimes 1$ for some unitaries $u_{1}(g, h) \in \mathscr{N}$. A direct computation shows that $\beta_{g}\left(u_{1}(h, k)\right) u_{1}(g, h k)=u_{1}(g, h) u_{1}(g h, k)$ for all $g, h, k \in G$.

Using 2.1.3 again, we find a (Borel) family $g \rightarrow v(g)$ of unitaries in $\mathscr{Q} \otimes M_{n}(\mathbb{C})$ with $u_{1}(g, h) \otimes 1=\left(\beta_{g} \otimes \iota\right)(v(h)) v(g) v(g h)^{*}$ for $g, h \in G$. Thus $w_{g} \alpha_{g}\left(w_{h}\right) u(g, h) w_{g h}^{*}$ $=w_{g} \alpha_{g}(v(h)) w_{g}^{*} v(g) v(g h)^{*}$ and so $u(g, h)=\alpha_{g}(d(h)) d(g) d(g h)^{*}$ where $d(g)=w_{g}^{*} v(g)$. Now

$$
\begin{aligned}
& \operatorname{Ad} d(g)^{*} \circ \alpha_{g} \circ \operatorname{Ad} d(h)^{*} \circ \alpha_{h} \\
= & \operatorname{Ad} d(g)^{*} \alpha_{g}\left(d(h)^{*}\right) \circ \alpha_{g} \circ \alpha_{h} \\
= & \operatorname{Ad} d(g)^{*} \alpha_{g}\left(d(h)^{*}\right) u(g, h) \circ \alpha_{g h} \\
= & \operatorname{Ad} d(g h)^{*} \circ \alpha_{g h},
\end{aligned}
$$


so that $g \rightarrow \operatorname{Ad} d(g)^{*} \circ \alpha_{g}$ is a splitting map for $\theta$.

Q.E.D.

Remark: The situation with $\mathscr{M}$ a $\mathrm{II}_{1}$-algebra (even the hyperfinite factor) and $G$ infinite seems to be unknown, except in trivial cases (e.g. $G=\mathbb{Z}$ ). Of particular interest is the case $G=Z^{2}$ where the question becomes: if $\alpha$ and $\beta$ commute modulo $\operatorname{Int}(\mathscr{M})$, are there unitaries $u, v \in \mathscr{M}$ with $\operatorname{Ad} u \circ \alpha$ and $\operatorname{Ad} v \circ \beta$ commuting?

\subsection{Comparison of Splitting Maps}

Let $\{\mathscr{M}, \theta\}$ be a split $G$-kernel, and $\alpha, \beta: G \rightarrow \operatorname{Aut}(\mathscr{M})$ be two splitting homomorphisms. Since $\varepsilon \circ \alpha=\varepsilon \circ \beta=\theta$, there is a Borel map $g \in G \rightarrow u_{g} \in \mathcal{U}(\mathscr{M})$ with $\alpha_{g}=\operatorname{Ad} u_{g} \circ \beta_{g}$. Since both $\alpha$ and $\beta$ are homomorphisms, we see, comparing $\alpha_{g} \circ \alpha_{h}$ with $\alpha_{g h}$, that $\mathrm{Ad} u_{g} \beta_{g}\left(u_{h}\right)=\mathrm{Ad} u_{g h}$ for all $g, h \in G$. We set $\omega(g, h)$ $=u_{g} \beta_{g}\left(u_{h}\right) u_{g h}^{*}$; evidently $(g, h) \rightarrow \omega(g, h)$ is Borel, and $\omega(g, h) \in \mathcal{U}(\mathcal{Z}(\mathscr{M})$ ). (In fact $\omega=\partial_{\alpha} u$ in the notations of 2.1.2, $\partial_{\alpha}$ signifying the boundary with respect to $\alpha$.)

Proposition 4.2.1. Let $\{\mathscr{M}, \theta\}, \alpha, \beta$ and $\omega$ be as above. Then

i) $\omega \in Z_{\theta}^{2}(G ; \mathcal{U}(\mathscr{Z}(\mathscr{M})))$, and the class of $\omega$ in $H_{\theta}^{2}(G ; \mathcal{U}(\mathscr{Z}(\mathscr{M})))$ depends only on $\alpha, \beta$. (We denote this class by $\left.\omega_{(\alpha, \beta)}\right)$

ii) If $r: G \rightarrow \operatorname{Aut}(\mathscr{M})$ is a third splitting map for $\{\mathscr{M}, \theta\}, \omega_{(\alpha, \beta)} \omega_{(\beta, \gamma)}$ $=\omega_{(\infty, \gamma)}$.

iii) $\omega_{(\alpha, \beta)}$ is trivial if and only if $\alpha$ and $\beta$ are exteriorly equivalent (see $[3 ; \S 2.2]$.

Proof. (i) Let $\omega(g, h)=u_{g} \beta_{g}\left(u_{h}\right) u_{g h}^{*}$ as above. Then

$$
\begin{aligned}
& \beta_{g}(\omega(h, k)) \omega(g, h k) \\
= & \beta_{g}\left(u_{h} \beta_{h}\left(u_{k}\right) u_{h k}^{*}\right) u_{g} \beta_{g}\left(u_{h k}\right) u_{g h k}^{*} \\
= & u_{g} \beta_{g}\left(u_{h}\right) \beta_{g h}\left(u_{k}\right) u_{g h k}^{*} \\
= & u_{g} \beta_{g}\left(u_{h}\right) u_{g h}^{*} u_{g h} \beta_{g h}\left(u_{k}\right) u_{g h k}^{*} \\
= & \omega(g, h) \omega(g h, k), \text { for all } g, h, k \in G .
\end{aligned}
$$

Thus $\omega \in Z_{\theta}^{2}(G ; \mathcal{U}(\mathscr{Z}(\mathscr{M})))$.

If $g \rightarrow u_{g}^{\prime}$ is another Borel map with $\alpha_{g}=\mathrm{Ad} u_{g}^{\prime} \circ \beta_{g}$, then, with $b_{g}=\left(u_{g}^{\prime}\right)^{*} u_{g}$ $=u_{g}\left(u_{g}^{\prime}\right)^{*} \in \mathcal{U}(\mathcal{Z}(\mathscr{M}))$, we see

$$
\begin{aligned}
u_{g} \beta_{g}\left(u_{h}\right) u_{g h}^{*} & =b_{g} u_{g}^{\prime} \beta_{g}\left(b_{h} u_{h}^{\prime}\right) b_{g h}^{*}\left(u_{g h}^{\prime}\right)^{*} \\
& =(\partial b)(g, h) u_{g}^{\prime} \beta_{g}\left(u_{h}^{\prime}\right)\left(u_{g h}^{\prime}\right)^{*}
\end{aligned}
$$


so that the 2-cocycles derived from $g \rightarrow u_{g}$ and $g \rightarrow u_{g}^{\prime}$ are cohomologous as required.

(ii) If $\alpha_{g}=\mathrm{Ad} u_{g} \circ \beta_{g}$ and $\beta_{g}=\mathrm{Ad} v_{g} \circ \gamma_{g}$, then $\alpha_{g}=\mathrm{Ad} u_{g} v_{g} \circ \gamma_{g}$, and

$$
\begin{aligned}
& u_{g} v_{g} r_{g}\left(u_{h} v_{h}\right) v_{g h}^{*} u_{g h}^{*} \\
= & u_{g} \beta_{g}\left(u_{h}\right) \beta_{g}\left(v_{h}\right) v_{g} v_{g h}^{*} u_{g h}^{*} \\
= & u_{g} \beta_{g}\left(u_{h}\right) v_{g} r_{g}\left(v_{h}\right) v_{g h}^{*} u_{g h}^{*} \\
= & \omega(g, h) \nu(g, h)
\end{aligned}
$$

where $\omega(g, h)=u_{g} \beta_{g}\left(u_{h}\right) u_{g h}^{*}, \nu(g, h)=v_{g} \gamma_{g}\left(v_{h}\right) v_{g h}^{*} ; g, h \in G$.

(iii) is trivial and left to the reader.

Q.E.D.

It follows from 4.2 .1 (iii) that if $\mathscr{M}$ is a factor and $H^{2}(G ; T)=(0)$ (e.g. $G=\boldsymbol{R}$-see [16]), then representations $\alpha, \beta$ of $G$ in $\operatorname{Aut}(\mathscr{M})$ are exteriorly equivalent if and only if $\varepsilon \circ \alpha=\varepsilon \circ \beta$ (c.f. [3]). We now investigate which elements of $H^{2}$ are of the form $\omega_{(\alpha, \beta)}$.

Theorem 4.2.2. Let $\{\mathscr{M}, \theta\}$ be a G-kernel, with splitting map $\alpha$. Then if either $\mathscr{M}$ is properly infinite, or $\mathscr{M}$ is a $I_{1}$-algebra and $G$ is finite, every element of $H_{\theta}^{2}(G ; \mathcal{U}(\mathcal{Z}(\mathscr{M})))$ is of the form $\omega_{(\alpha, \beta)}$ for some splitting map $\beta$.

Proof. Let $\omega \in Z_{\theta}^{2}(G ; \mathcal{U}(\mathcal{Z}(\mathscr{M})))$ be arbitrary. In either case, it is sufficient to show there is a Borel map $g \rightarrow u_{g} \in \mathcal{U}(\mathscr{M})$ with $\omega(g, h)=\alpha_{g}\left(u_{h}\right) u_{g} u_{g h}^{*}$, for then, with $\beta_{g}=\operatorname{Ad} u_{g}^{*} \circ \alpha_{g}$ we see

$$
\begin{aligned}
\beta_{g} \circ \beta_{h} & =\operatorname{Ad} u_{g}^{*} \alpha_{g}\left(u_{h}^{*}\right) \circ \alpha_{g h} \\
& =\operatorname{Ad} u_{g h}^{*} \omega(g, h)^{*} \circ \alpha_{g h} \\
& =\beta_{g h} .
\end{aligned}
$$

Thus $\beta$ is a splitting map for $\theta$, and by construction $\omega=\omega_{(\omega, \beta)}$. As in the proof of 4.1.3, we argue the two situations separately.

Case 1: $\mathscr{M}$ properly infinite.

Let $M_{\infty}(\boldsymbol{C})$ denote the $\mathrm{I}_{\infty}$-factor. From the proof of Lemma 4.7 of [31] (see also proof of 4.1.3) we may choose an isomorphism $\sigma: \mathscr{M} \rightarrow \mathscr{M} \otimes M_{\infty}(C)$ and a Borel map $g \rightarrow w_{g} \in \mathcal{U}\left(\mathscr{H} \otimes M_{\infty}(\boldsymbol{C})\right)$ with $\alpha_{g} \otimes_{\iota}=\mathrm{Ad} w_{g} \circ \sigma \circ \alpha_{g} \circ \sigma^{-1}$ and $w_{g} \gamma_{g}\left(w_{h}\right)=w_{g h}$, where $\gamma_{g}=\sigma \circ \alpha_{g} \circ \sigma^{-1}$. By construction, $\sigma(\alpha)=\alpha \otimes 1$ for $a \in$ $\mathscr{Z}(\mathscr{M})$.

From 2.1.3, there is a Borel map $g \rightarrow v(g) \in \mathcal{U}\left(\mathscr{M} \otimes M_{\infty}(\mathbb{C})\right)$ with

$$
\omega(g, h) \otimes 1=\left(\alpha_{g} \otimes \iota\right)(v(h)) v(g) v(g h)^{*} ; g, h \in G .
$$


Thus

$$
\begin{aligned}
\omega(g, h)^{*} \otimes 1 & =v(g)^{*}\left(\alpha_{g} \otimes \iota\right)\left(v(h)^{*}\right) v(g h) \\
& =v(g)^{*} w_{g} \gamma_{g}\left(v(h)^{*}\right) w_{g}^{*} v(g h) \\
& =v(g)^{*} w_{g} \gamma_{g}\left(v(h)^{*} w_{h}\right) w_{g h}^{*} v(g h) \\
& =c(g) r_{g}(c(h)) c(g h)^{*} \quad \text { where } \quad c(g)=v(g)^{*} w_{g} .
\end{aligned}
$$

Now

$$
\begin{aligned}
\omega(g, h)^{*} & =\sigma^{-1}\left(\omega(g, h)^{*} \otimes \iota\right) \\
& =\sigma^{-1}\left(c(g) \sigma \circ \alpha_{g} \circ \sigma^{-1}(c(h)) c(g h)^{*}\right) \\
& =d(g) \alpha_{g}(d(h)) d(g h)^{*} \quad \text { where } \quad d(g)=\sigma^{-1}(c(g)) .
\end{aligned}
$$

So with $u_{g}=d(g)^{*}$ we obtain

$$
\omega(g, h)=\alpha_{g}\left(u_{h}\right) u_{g} u_{g h}^{*} \quad \text { as required. }
$$

Case 2: $\mathscr{M}$ a $\mathrm{II}_{1}$-algebra, $G$ has order $n$.

We adopt the notation of the proof of 4.2.3, Case 2. Thus there are unitaries $w_{g} \in \mathscr{Q} \otimes M_{n}(\boldsymbol{C})$ and automorphisms $\beta_{g}, g \in G$ of $\mathscr{n}$ with $\operatorname{Ad} w_{g} \circ \alpha_{g}$ $=\beta_{g} \otimes \ell$, where $\mathscr{M}$ is identified with $\mathscr{R} \otimes M_{n}(\boldsymbol{C})$. There seems to be no easy guarantee that $g \rightarrow \beta_{g}$ is a representation; however, as computed in 4.1.3, the obstruction associated to $g \rightarrow \varepsilon\left(\beta_{g}\right)$ is trivial. Thus, from the conclusion of 4.1.3, we may choose unitaries $w_{g}^{\prime} \in \mathscr{M}$ with $g \rightarrow \operatorname{Ad} w_{g}^{\prime} \circ \beta_{g}$ a representation of $G$. By changing $w_{g}$ above to $\left(w_{g}^{\prime} \otimes 1\right) w_{g}$, we may assume that $g \rightarrow \beta_{g}$ is a representation of $G$ in $\operatorname{Aut}(\mathscr{M})$.

Since $\omega(g, h) \in \mathscr{L}(\mathscr{M})$, we may write $\omega(g, h)=\nu(g, h) \otimes 1$, with $\nu(g, h) \in \mathscr{L}(\mathscr{I})$; clearly $(g, h) \rightarrow \nu(g, h)$ is a $\beta$-2-cocycle. Since both $g \rightarrow \alpha_{g}$ and $g \rightarrow \beta_{g}$ are representations, we see that $w_{g} \alpha_{g}\left(w_{h}\right) w_{g h}^{*}$ is central; we write $w_{g} \alpha_{g}\left(w_{h}\right) w_{g h}^{*}=\mu(g, h) \otimes 1$ with $\mu(g, h) \in \mathcal{U}(\mathcal{Z}(\mathscr{R}))$. It is readily checked that $(g, h) \rightarrow \mu(g, h)$ is also a $\beta$-2-cocycle. Thus from 2.1.3, there are unitaries $b(g) \in \mathscr{R} \otimes M_{n}(\boldsymbol{C})$ with

$$
\begin{aligned}
\nu(g, h) \mu(g, h) \otimes 1 & =\left(\beta_{g} \otimes \iota\right)(b(h)) b(g) b(g h)^{*} \\
& =w_{g} \alpha_{g}(b(h)) w_{g}^{*} b(g) b(g h)^{*} .
\end{aligned}
$$

Thus

$$
\omega(g, h) w_{g} \alpha_{g}\left(w_{h}\right) w_{g h}^{*}=w_{g} \alpha_{g}(b(h)) w_{g}^{*} b(g)(g h)^{*}
$$

and

$$
\omega(g, h)=\alpha_{g}(u(h)) u(g) u(g h)^{*}, h, g \in G,
$$

where $u(g)=w_{g}^{*} b(g)$. Thus $\omega$ has the required form.

Q.E.D. 


\subsection{Regularization of Extensions}

Let $\{\mathscr{M}, \theta\}$ be a split $G$-kernel, and $\alpha$ a fixed splitting map; let $\{\Re, I, \pi\}$ be an extension of $\mathscr{M}$ by $G$ realizing $\theta$. Define automorphisms $\beta_{g}, g \in G$ of $\mathscr{M}$, and unitaries $v(g, h) \in \mathscr{M}, g, h \in G$ by

$$
\begin{aligned}
& I\left(\beta_{g}(x)\right)=\pi(g) I(x) \pi(g)^{*} ; x \in \mathscr{M}, g \in G ; \\
& \pi(g) \pi(h)=I(v(g, h)) \pi(g h) ; g, h \in G .
\end{aligned}
$$

Clearly $g \rightarrow \beta_{g}$ and $(g, h) \rightarrow v(g, h)$ are Borel maps. Since $\varepsilon \circ \alpha=\varepsilon \circ \beta=\theta$, there is a Borel map $g \in G \rightarrow u_{g} \in \mathcal{U}(\mathscr{M})$ with $\operatorname{Ad} u_{g} \circ \beta_{g}=\alpha_{g}$. Thus

$$
\begin{aligned}
I\left(u_{g}\right) \pi(g) I\left(u_{h}\right) \pi(h) & =I\left(u_{g} \beta_{g}\left(u_{h}\right) v(g, h)\right) \pi(g h) \\
& =I\left(u_{g} \beta_{g}\left(u_{h}\right) v(g, h) u_{g h}^{*}\right) I\left(u_{g h}\right) \pi(g h) .
\end{aligned}
$$

Also, with $\pi_{1}(g)=I\left(u_{g}\right) \pi(g)$ we see

$$
\begin{aligned}
\pi_{1}(g) I(x) \pi_{1}(g)^{*} & =I\left(u_{g} \beta_{g}(x) u_{g}^{*}\right) \\
& =I\left(\alpha_{g}(x)\right), x \in \mathscr{M} .
\end{aligned}
$$

Since $\alpha_{g} \circ \alpha_{h}=\alpha_{g h}$, we see $\omega(g, h)=u_{g} \beta_{g}\left(u_{h}\right) v(g, h) u_{g h}^{*}$ is central in $\mathscr{M}$. Also, we may compute

$$
\begin{aligned}
& I\left(\alpha_{g}(\omega(h, k)) \omega(g, h k)\right) \\
= & \pi_{1}(g) \pi_{1}(h) \pi_{1}(k) \pi_{1}(h k)^{*} \pi_{1}(g)^{*} \pi_{1}(g) \pi_{1}(h k) \pi_{1}(g h k)^{*} \\
= & \pi_{1}(g) \pi_{1}(h) \pi_{1}(k) \pi_{1}(g h k)^{*} \\
= & \pi_{1}(g) \pi_{1}(h) \pi_{1}(g h)^{*} \pi_{1}(g h) \pi_{1}(k) \pi_{1}(g h k)^{*} \\
= & I(\omega(g, h) \omega(g h, k)) \quad \text { for } g, h, k \in G .
\end{aligned}
$$

Thus $\omega \in Z_{\theta}^{2}(G ; \mathcal{U}(\mathcal{Z}(\mathscr{M}))$ ). If we consider $\alpha$ and $\{\mathscr{l}, I, \pi\}$ as given data, the only indeterminacy in constructing $\omega$ is in the choice of unitaries $u_{g}$ with Ad $u_{g} \circ \beta_{g}=\alpha_{g}$. However if $g \rightarrow u_{g}^{\prime}$ is another Borel map with $\operatorname{Ad} u_{g}^{\prime} \circ \beta_{g}=\alpha_{g}$, the 2-cocycle $\omega^{\prime}(g, h)=u_{g}^{\prime} \beta_{g}\left(u_{h}^{\prime}\right) v(g, h) u_{g h}^{\prime}$ is cohomologous to $\omega$; clearly also any 2-cocycle cohomologous to $\omega$ arises in this way. Finally, it is trivial to check that we may choose $\pi_{1}$ to have the properties $\pi_{1}(g) \in I(\mathscr{M}) \pi(g), \pi_{1}(g) I(x)$ $\times \pi_{1}(g)^{*}=I\left(\alpha_{g}(x)\right)$ and $\pi_{1}(g) \pi_{1}(h)=\pi_{1}(g h)$ for $g, h \in G, x \in \mathscr{M}$, if and only if the 2-cocycle $\omega$ associated to $\{\Re, I, \pi\}$ and $\alpha$ is (cohomologically) trivial. We record this as

Proposition 4.3.1. Let $\{\mathscr{M}, \theta\}$ be a split G-kernel and $\alpha$ a fixed splitting map. Let $\{\mathscr{N}, I, \pi\}$ be an extension of $\mathscr{M}$ be $G$ realizing $\theta$. Then there is a 
2-cocycle $\omega \in Z_{\theta}^{2}(G ; \mathcal{U}(\mathcal{Z}(\mathscr{M})))$ and an extension $\left\{\mathscr{N}, I, \pi_{1}\right\}$ of $\mathscr{M}$ by $G$ realizing $\theta$ with

(i) $\operatorname{Ad} \pi_{1}(g)(I(x))=I\left(\alpha_{g}(x)\right) ; g \in G, x \in \mathscr{M}$,

(ii) $\pi_{1}(g) \pi_{1}(h)=I(\omega(g, h)) \pi_{1}(g h) ; g, h \in G$,

(iii) $\pi_{1}(g) \in I(\mathscr{M}) \pi(g)$.

The cohomology class of $\omega$ is uniquely determined by $\alpha$ and $\{\Re, I, \pi\}$, and is trivial if and only if $\pi_{1}$ may be chosen to be a representation.

An extension $\{\Re, I, \pi\}$ satisfying (i), (ii) and (iii) of 4.3 .1 will be said to have been regularized, or normalized. Although 4.3.1 is to be expected, it is unsatisfactory in many respects. First, trivial examples show that the class of $\omega$ does not determine $\Re$. If we denote the unique cohomology class in $H_{\theta}^{2}(G ; \mathcal{U}(\mathscr{Z}(\mathscr{M})))$ determined by 4.3 .1 by $\omega(\Re, I, \pi, \alpha)$, it seems quite likely that there are extensions $\left\{\mathscr{R}, I, \pi_{1}\right\}$ and $\left\{\mathscr{N}, I, \pi_{2}\right\}$ of $\mathscr{M}$ by $G$ realizing a fixed homomorphism $\theta: G \rightarrow \operatorname{Out}(\mathcal{M})$, but $\omega\left(\mathscr{I}, I, \pi_{1}, \alpha\right) \neq \omega\left(\mathscr{R}, I, \pi_{2}, \alpha\right)$. This of course will not happen if $I(\mathscr{M})^{\prime} \cap \mathscr{I} \subseteq I(\mathscr{M})$, for then $\pi_{2}(g) \in I(\mathscr{M}) \pi_{1}(g)$, $g \in G$. The above relative commutant condition is known to hold (e.g.) if $\mathscr{M}$ is abelian, $G$ is countable and acts freely, and $\mathscr{N}$ is the twisted crossed product. (See [32], and [31] for an extensive discussion of this condition.) The problem of whether or not $\omega\left(\mathscr{N}, I_{1}, \pi_{1}, \alpha\right)=\omega\left(\mathscr{R}, I_{2}, \pi_{2}, \alpha\right)$ in case $\mathscr{M}$ is abelian, $G$ is countable and acts freely, and $\Re$ is a twisted crossed product is directly related to the uniqueness or otherwise of regular maximal abelian subalgebras of $\mathcal{n}$.

It is, however, easy to describe the dependence of $\omega(\eta, I, \pi, \alpha)$ on $\alpha$.

Proposition 4.3.2. Let $\{\mathscr{M}, \theta\}$ be a split G-kernel, let $\alpha, \beta$ be two splitting maps, and let $\{\mathscr{R}, I, \pi\}$ be an extension of $\mathscr{M}$ by $G$ realizing $\theta$. Then $\omega(\mathcal{N}, I$, $\pi, \beta)=\omega_{(\beta, \alpha)} \omega(\Re, I, \pi, \alpha)$, where $\omega_{(\beta, \alpha)}$ is as in 4.2.1.

Proof. Choose Borel maps $\pi_{\alpha}, \pi_{\beta}: G \rightarrow \mathcal{U}(\mathscr{l})$ satisfying the conclusions of 4.3.1 for $\alpha$ and $\beta$ respectively. Write $\pi_{\alpha}(g) \pi_{\alpha}(h)=I\left(\omega_{\alpha}(g, h)\right) \pi_{\alpha}(g h)$ and $\pi_{\beta}(g) \pi_{\beta}(h)=I\left(\omega_{\beta}(g, h)\right) \pi_{\beta}(g h)$ for $g, h \in G$. Since both $\pi_{\omega}(g)$ and $\pi_{\beta}(g)$ are in $I(\mathscr{M}) \pi(g)$, there is a Borel map $g \rightarrow u_{g} \in \mathcal{U}(\mathscr{M})$ with $\pi_{a}(g)=I\left(u_{g}\right) \pi_{\beta}(g), g \in G$. Thus $\alpha_{g}=\operatorname{Ad~} u_{g} \circ \beta_{g}$. Also

so that

$$
\begin{aligned}
\pi_{\beta}(g) \pi_{\beta}(h) & =I\left(u_{g}^{*}\right) \pi_{\alpha}(g) I\left(u_{h}^{*}\right) \pi_{\alpha}(h) \\
& =I\left(u_{g}^{*} \alpha_{g}\left(u_{h}^{*}\right) \omega_{\alpha}(g, h) u_{g h}\right) \pi_{\beta}(g h)
\end{aligned}
$$

However the classes of $\omega_{\alpha}, \omega_{\beta}$ and $(g, h) \rightarrow u_{g}^{*} \alpha_{g}\left(u_{h}^{*}\right) u_{g h}$ in $H_{\theta}^{2}(G ; \mathcal{U}(\mathcal{Z}(\mathscr{M})))$ 
are, respectively, $\omega(\Re, I, \pi, \alpha), \omega(\Re, I, \pi, \beta)$ and $\omega_{(\beta, \alpha)}$, as required. Q.E.D.

We now obtain

Theorem 43.3. Let $\{\mathscr{M}, \theta\}$ be a G-kernel with trivial obstruction, $\{\Re, I, \pi\}$ an extension of $\mathscr{M}$ by $G$ realizing $\theta$. Suppose further $\mathscr{M}$ is either properly infinite, or $\mathscr{M}$ is a $I I_{1}$-algebra, and $G$ is finite; then there is an extension $\left\{\mathscr{R}, I_{1}, \pi_{1}\right\}$ of $\mathscr{M}$ by $G$ realizing $\theta$ with

a) $\pi_{1}(g) \pi_{1}(h)=\pi_{1}(g h) ; g, h \in G$,

b) $\pi_{1}(g) \in I(\mathscr{M}) \pi(g), g \in G$.

Proof. From 4.1.3, $\{\mathscr{M}, \theta\}$ is split; we let $\alpha$ be an arbitrary splitting map, and $\omega=\omega(\mathfrak{I}, I, \pi, \alpha)$. From 4.2.2, $\omega=\omega_{(\alpha, \beta)}$ for some splitting map $\beta$ for $\{\mathscr{M}, \theta\}$. From 4.3.2 $\omega(\mathscr{M}, I, \pi, \beta)=\omega_{(\beta, \alpha)} \omega=\omega^{-1}$. $\omega$ is trivial. The desired map $\pi_{1}$ may now be found by virtue of 4.3.1.

Q.E.D.

The above result should be regarded as a complement of [27; Theorem 5.1].

The extension theory of properly infinite von Neumann algebras, or of $\mathrm{II}_{1}$-algebras by finite groups, is thus particularly simple from an algebraic point of view, even though one is obliged to deal with (possibly) of a multiplicity of liftings for a given kernel. Our results also have significance for injective von Neumann algebras ([7]) and for the representation theory of certain locally compact groups, as will be seen in Section 6.

\section{$\S 5$. Isomorphism Criteria for Extensions}

Here, we seek criteria on an extension $\{\mathscr{N}, I, \pi\}$ of $\mathscr{M}$ by $G$ in order that it be equivalent (in a sense made precise below) to a twisted crossed product extension.

\subsection{Algebraic Criteria}

There are several notions of equivalence for extensions of $\mathscr{M}$ by $G$ which are amenable to interpretation in our context.

Definition 5.1.1. Extensions $\left\{\mathscr{I}_{1}, I_{1}, \pi_{1}\right\}$ and $\left\{\mathscr{I}_{2}, I_{2}, \pi_{2}\right\}$ of $\mathscr{M}$ by $G$ are $\mathscr{M}-G$ equivalent if there is a normal isomorphism $\kappa: \mathscr{I}_{1} \rightarrow \mathscr{I}_{2}$ with

a) $\quad \kappa\left(I_{1}(\mathscr{M})\right)=I_{2}(\mathscr{M})$

b) $\kappa\left(\pi_{1}(g)\right) \in I_{2}(\mathscr{M}) \pi_{2}(g), g \in G$. 
If $\kappa$ may be chosen so that $\kappa\left(I_{1}(x)\right)=I_{2}(x), x \in \mathscr{M}$ we say the extensions are strongly $\mathscr{M}$-G-equivalent; if, in addition $\kappa\left(\pi_{1}(g)\right)=\pi_{2}(g)$ we say $\left\{\mathscr{\Omega}_{1}, I_{1}, \pi_{1}\right\}$ and $\left\{\mathscr{I}_{2}, I_{2}, \pi_{2}\right\}$ are isomorphic as extensions.

It should be noted that the modifications of extensions carried out in 4.3.1 and 4.3.3 have taken place within a single strong equivalence class.

Proposition 5.1.2. Let $\left\{\mathscr{I}_{j}, I_{j}, \pi_{j}\right\}, j=1,2$ be extensions of $\mathscr{M}$ by $G$, and write

$$
\operatorname{Ad} \pi_{j}(g)\left(I_{j}(x)\right)=I_{j}\left(\alpha_{g}^{j}(x)\right) ; j=1,2, g \in G, x \in \mathscr{M}
$$

and

$$
\pi_{j}(g) \pi_{j}(h)=I_{j}\left(v_{j}(g, h)\right) \pi_{j}(g, h) ; j=1,2, g, h \in G .
$$

Then, if $\left\{\mathscr{l}_{1}, I_{1}, \pi_{1}\right\}$ and $\left\{\mathscr{I}_{2}, I_{2}, \pi_{2}\right\}$ are $\mathscr{M}$-G-equivalent (resp. strongly $\mathscr{M}$-Gequivalent), there exists $\sigma \in \operatorname{Aut}(\mathscr{M})$ (resp. $\sigma=\imath$ ) and a Borel map $g \in G \rightarrow u_{g} \in$ $\vartheta(\mathscr{M})$ with

$$
v_{2}(g, h)=\alpha_{g}^{2}\left(u_{h}^{*}\right) u_{g}^{*} \sigma\left(w_{1}(g, h)\right) u_{g h} ; g, h \in G
$$

and

$$
\alpha_{g}^{2}=\operatorname{Ad} u_{g}^{*} \circ \sigma \circ \alpha_{g}^{1} \circ \sigma^{-1} ; g \in G .
$$

The converse holds if the extensions $\left\{\bigcap_{j}, I_{j}, \pi_{j}\right\}, j=1,2$ are regular in the sense of 3.1 .

Proof. Suppose the extensions are (strongly) equivalent, and choose an isomorphism $\kappa: \mathscr{I}_{1} \rightarrow \mathscr{I}_{2}$ with $\kappa\left(I_{1}(\mathscr{M})\right)=I_{2}(\mathscr{M}), \kappa\left(\pi_{1}(g)\right) \in I_{2}(\mathscr{M}) \pi_{2}(g)$. Define $\sigma$ and $g \rightarrow u_{g}$ by

$$
\kappa\left(I_{1}(x)\right)=I_{2}(\sigma(x)), x \in \mathscr{M}
$$

and

$$
\kappa\left(\pi_{1}(g)\right)=I_{2}\left(u_{g}\right) \pi_{2}(g), g \in G .
$$

Note that in the case of strong equivalence, we may choose $\sigma=\imath$. The indicated relations follow trivially by applying $\kappa$ to the equations

$$
\pi_{1}(g) \pi_{1}(h)=I_{1}\left(v_{1}(g, h)\right) \pi_{1}(g h) ; g, h \in G ;
$$

and

$$
\pi_{1}(g) I_{1}(x) \pi_{1}(g)^{*}=I_{1}\left(\alpha_{g}^{1}(x)\right) ; x \in \mathscr{M}, g \in G ;
$$

and using the definitions of $v_{2}(g, h), \sigma$ and $u_{g}$.

Conversely, suppose the extensions $\left\{\bigcap_{j}, I_{j}, \pi_{j}\right\}, j=1,2$ are regular and 
$\alpha_{g}^{j}, v_{j}(g, h), \sigma, u_{g}$ satisfy the relations above. We may assume that $\mathscr{M}$ acts on $\mathcal{H}$, that $\mathscr{\eta}_{j}, j=1,2$ act on $L^{2}(G ; \mathcal{H})$ and $I_{j}, \pi_{j}$ are given by

$$
\begin{aligned}
& \left(I_{j}(x) \xi\right)(g)=\alpha_{g-1}^{j}(x) \xi(g), x \in \mathscr{M} ; \\
& \left(\pi_{j}(h) \xi\right)(g)=v_{j}\left(g^{-1}, h\right) \xi(g), h \in G ;
\end{aligned}
$$

for $j=1,2$ and $\xi \in L^{2}(G ; \mathcal{H})$. We may further assume that $\sigma$ is of the form Ad $U$ for some unitary $U$ on $\mathcal{H}$.

Define a unitary $V$ on $L^{2}(G ; \mathcal{H})$ by $(V \xi)(g)=u_{g-1}^{*} U \xi(g)$. We compute

$$
\begin{aligned}
\left(V I_{1}(x) V^{*} \xi\right)(g) & =u_{g^{-1}}^{*} U \alpha_{g^{-1}}^{1}(x) U^{*} u_{g^{-1}} \xi(g) \\
& =\operatorname{Ad} u_{g^{-1} \circ \sigma \circ \alpha_{g^{-1}}^{*} \circ \sigma^{-1}(\sigma(x)) \xi(g)} \\
& =\alpha_{g^{-1}}^{2}(\sigma(x)) \xi(g) \\
& =\left(I_{2}(\sigma(x)) \xi\right)(g) ; x \in \mathscr{M}, \xi \in L^{2}(G ; \mathcal{H})
\end{aligned}
$$

and

$$
\begin{aligned}
\left(V \pi_{1}(h) V^{*} \xi\right)(g) & =u_{g^{-1}}^{*} U v_{1}\left(g^{-1}, h\right) U^{*} u_{g^{-1}} \xi\left(h^{-1} g\right) \\
& =u_{g^{-1}}^{*} \sigma\left(v_{1}\left(g^{-1}, h\right)\right) u_{g^{-1}} \xi\left(h^{-1} g\right) \\
& =\alpha_{g^{-1}}^{2}\left(u_{h}\right) v_{2}\left(g^{-1}, h\right) \xi\left(h^{-1} g\right) \\
& =\alpha_{g^{-1}}^{2}\left(u_{h}\right)\left(\pi_{2}(h) \xi\right)(g) \\
& =\left(I_{2}\left(u_{h}\right) \pi_{2}(h) \xi\right)(g)
\end{aligned}
$$

In particular, $\mathscr{M}$-G-equivalence of the extensions $\left\{\mathscr{M}_{j}, I_{j}, \pi_{j}\right\} \quad j=1,2$ implies conjugacy of the corresponding homomorphisms $\theta^{j}: G \rightarrow \operatorname{Out}(\mathscr{M})$. Also, if $g \in G \rightarrow \alpha_{g} \in \operatorname{Aut}(\mathcal{M})$ is a fixed representation of $G$ as automorphisms, $\omega \in Z_{a}^{2}(G ; \mathcal{U}(\mathcal{Z}(\mathscr{M})))$ and $\sigma \in \operatorname{Aut}(\mathscr{M})$ commutes with $\left\{\alpha_{g}, g \in G\right\}$, then $\mathscr{R}(\mathscr{M}, G, \alpha, \omega)$ and $\mathscr{R}(\mathscr{M}, G, \alpha, \sigma \omega)$ (twisted crossed products) are $\mathscr{M}$ - $G$-equivalent and so certainly isomorphic. So in general (if $H^{2}(G ; \mathbb{T}) \neq\{0\}$ ) we may have $\mathscr{R}(\mathscr{M}, G, \alpha, \omega)$ isomorphic with $\mathscr{R}(\mathscr{H}, G, \alpha, \nu)$ but $\omega$ not cohomologous with $\nu$.

\subsection{Plancherel Pairs}

Throughout, $\{\mathscr{M}, \theta\}$ will denote a fixed split $G$-kernel, and $\alpha$ a fixed splitting map; since $\alpha$ is Borel and $G$ and $\operatorname{Aut}(\mathcal{M})$ both Polish, $\alpha$ is in fact continuous (see [2]). According to 4.3.1, each extension of $\mathscr{M}$ by $G$ realizing $\theta$ has a normalized form $\{\Re, I, \pi\}$ i.e.

$$
\begin{aligned}
& \text { Ad } \pi(g)(I(x))=I\left(\alpha_{g}(x)\right) ; x \in \mathcal{M}, g \in G, \\
& \pi(g) \pi(h)=I(\omega(g, h)) \pi(g h) ; g, h \in G,
\end{aligned}
$$


for some $\omega \in Z_{\theta}^{2}(G ; \mathcal{U}(\mathscr{Z}(\mathscr{M}))$ ). (For convenience we say $\{\mathscr{R}, I, \pi\}$ induces $\alpha$ and $\omega$ ). Thus $\{\mathscr{R}, I, \pi\}$ enjoys the same algebraic properties as the twisted product extension $\left\{\mathscr{R}(\mathscr{H}, G, \alpha, \omega), I^{\alpha}, \lambda^{\omega}\right\}$ (example 3.1.3 and [27]). We shall develop necessary and sufficient conditions for a normalized extension inducing $\alpha$ and $\omega$ to be isomorphic with a twisted crossed product extension.

In the following discussion, when we refer to a weight on a von Neumann algebra, we mean a faithful, normal, semifinite weight; we omit the qualifications. We refer to [29] for the theory of weights and left Hilbert algebras.

Let $\{\mathscr{N}, I, \pi\}$ be an arbitrary extension of $\mathscr{M}$ by $G$; for $x \in \mathcal{K}(G ; \mathscr{M})$, the space of norm-bounded Borel maps $x: g \in G \rightarrow x(g) \in \mathscr{M}$ with $\{g: x(g) \neq 0\}$ precompact, define an operator $\tilde{x} \in \mathscr{N}$ by $\tilde{x}=\int_{G} \pi(g) I(x(g)) d g$ (this exists as a weak integral).

Definition 5.2.1. In the above situation, a pair $\{\varphi, \tilde{\varphi}\}$ of weights is a Plancherel pair for $\{\mathscr{M}, \mathscr{N}, I, \pi\}$ if

(i) $\varphi$ is a weight on $\mathscr{M}, \tilde{\varphi}$ a weight on $\mathscr{R}$,

(ii) $\tilde{\mathfrak{B}}=\{y \in \mathscr{M}: y=\tilde{x}$ for some $x \in \mathcal{K}(G ; \mathscr{M})$ and

$$
\left.\widetilde{\varphi}\left(y^{*} y+y y^{*}\right)<\infty\right\}
$$

is dense, with respect to the \#-norm, in the full left Hilbert algebra $\tilde{\mathfrak{U}}$ determined by $\tilde{\varphi}$

(iii) for $x \in \mathcal{K}(G ; \mathscr{M})$ with $\tilde{x} \in \tilde{\mathfrak{B}}$

$$
\tilde{\varphi}\left(\tilde{x}^{*} \tilde{x}\right)=\int_{G} \varphi\left(x(g)^{*} x(g)\right) d g .
$$

Theorem 5.2.2. Let $\{\mathscr{M}, \theta\}, \alpha$, be as above, and $\omega \in Z_{\theta}^{2}(G ; \mathcal{U}(\mathcal{Z}(\mathscr{M})))$.

(i) $\{\mathscr{R}, I, \pi\}$ is $\mathscr{M}$-G-equivalent with $\left\{\mathscr{R}(\mathscr{M}, G, \alpha, \omega), I^{\alpha}, \lambda^{\omega}\right\}$ if and only if $\{\mathscr{M}, \mathscr{\Omega}, I, \pi\}$ admits a Plancherel pair of weights,

(ii) if $\{\Re, I, \pi\}$ is normalized, induces $\alpha$ and $\omega$, and admits a Plancherel pair of weights, the extensions $\{\mathscr{\eta}, I, \pi\}$ and $\left\{\mathscr{R}(\mathscr{M}, G, \alpha, \omega), I^{\alpha}, \lambda^{\omega}\right\}$ are isomorphic (as extensions).

Proof. (i) Let $\kappa: \mathscr{N} \rightarrow \mathscr{R}(\mathscr{M}, G, \alpha, \omega)$ be an isomorphism implementing the $\mathscr{M}$-G-equivalence of $\{\mathscr{N}, I, \pi\}$ and $\left\{\mathscr{R}(\mathscr{M}, G, \alpha, \omega), I^{\alpha}, \lambda^{\omega}\right\}$. We may thus suppose $\mathscr{I}=\mathscr{R}(\mathscr{M}, G, \alpha, \omega), I(x)=I^{\alpha}(\sigma(x)), x \in \mathscr{M}$ and $\pi(g)=\lambda^{\omega}(g) I^{\omega}\left(u_{g}\right)$, $g \in G$, for some $\sigma \in \operatorname{Aut}(\mathscr{M})$ and Borel map $g \rightarrow u_{g} \in \mathcal{U}(\mathscr{M})$.

Let $\varphi$ be a weight on $\mathscr{M}$, and $\tilde{\varphi}$ the weight on $\mathscr{N}=\mathscr{R}(\mathscr{M}, G, \alpha, \omega)$ dual to $\psi=\varphi \circ \sigma^{-1}$ (see [27]). Noting that for $x \in \mathcal{K}(G ; \mathscr{M})$ we have 


$$
\begin{aligned}
\tilde{x} & =\int_{G} \pi(g) I(x(g)) d g \\
& =\int_{G} \lambda^{\omega}(g) I^{\omega}\left(u_{g} \sigma(x(g)) d g,\right.
\end{aligned}
$$

so that

$$
\begin{aligned}
\tilde{\varphi}\left(\tilde{x}^{*} \tilde{x}\right) & =\int_{G} \psi\left(\left(u_{g} \sigma(x(g))\right)^{*} u_{g} \sigma(x(g))\right) d g \\
& =\int_{G} \varphi\left(x(g)^{*} x(g)\right) d g,
\end{aligned}
$$

so that $\{\varphi, \tilde{\varphi}\}$ is a Plancherel pair for $\{\mathscr{M}, \mathscr{I}, I, \pi\}$. (The other requirements of the definition are easily verified using [27].)

Conversely, suppose that $\{\mathscr{M}, \mathscr{I}, I, \pi\}$ admits a Plancherel pair of weights $\{\varphi, \tilde{\varphi}\}$, and let $\left\{\mathscr{I}, I, \pi_{1}\right\}$ be the normalized form of $\{\mathscr{l}, I, \pi\}$, so that $\left\{\mathscr{l}, I, \pi_{1}\right\}$ induces $\alpha$ and $\omega$. We claim that $\{\varphi, \tilde{\varphi}\}$ is a Plancherel pair for $\left\{\mathscr{M}, \mathscr{N}, I, \pi_{1}\right\}$.

Note that if $x \in \mathcal{K}(G ; \mathscr{M})$, and we write $\pi(g)=\pi_{1}(g) I\left(u_{g}\right)$, then

$$
\begin{aligned}
\tilde{x} & =\int_{G} \pi(g) I(x(g)) d g \\
& =\int_{G} \pi_{1}(g) I\left(u_{g} x(g)\right) d g .
\end{aligned}
$$

Thus

$$
\begin{aligned}
\tilde{\varphi}\left(\tilde{x}^{*} \tilde{x}\right) & =\int_{G} \varphi\left(x(g)^{*} x(g)\right) d g \\
& =\int_{G} \varphi\left(\left(u_{g} x(g)\right)^{*} u_{g} x(g)\right) d g
\end{aligned}
$$

and $\{\varphi, \tilde{\varphi}\}$ satisfies 5.2 .1 (iii) for $\left\{\mathscr{M}, \mathscr{N}, I, \pi_{1}\right\}$. Using this, 5.2.1. (ii) is trivially verified. To complete the proof then it is sufficient to prove 5.2.2 (ii).

(ii) Let $\{\mathscr{N}, I, \pi\}$ be a normalized extension of $\mathscr{M}$ by $G$ inducing $\alpha$ and $\omega$, and let $\{\varphi, \tilde{\varphi}\}$ be a Plancherel pair for $\{\mathscr{H}, \mathscr{N}, I, \pi\}$. We suppose that $\mathscr{M}$ (resp. $\mathscr{R}$ ) acts on the Hilbert space $\mathscr{H}$ (resp. $\widetilde{\mathcal{H}}$ ) derived from $\varphi$ (resp. $\tilde{\varphi}$ ), and we let $\Lambda$, (resp. $\tilde{\Lambda})$ denote the canonical map from the full left Hilbert algebra $\mathfrak{U}($ resp. $\tilde{\mathfrak{U}})$ derived from $\varphi$ (resp. $\tilde{\varphi}$ ) into $\mathscr{H}$ (resp. $\widetilde{\mathcal{H}}$ ). By hypothesis, $\tilde{\Lambda}(\widetilde{\mathfrak{B}})$ is dense in $\tilde{\mathcal{H}}$.

Note that for $x \in \mathcal{K}(G ; \mathscr{M})$ with $\tilde{x} \in \tilde{\mathfrak{B}}$, then $\tilde{x}=0$ implies $0=\tilde{\varphi}\left(\tilde{x}^{*} \tilde{x}\right)$ $=\int_{G} \varphi\left(x(g)^{*} x(g)\right) d g$, so that $x(g)=0$ a.e. Thus there is a well defined linear map $U: \tilde{\Lambda}(\widetilde{\mathfrak{B}}) \rightarrow L^{2}(G ; \mathcal{H})$ given by $(U(\tilde{\Lambda}(\tilde{x})))(g)=\Lambda\left(\omega\left(g^{-1}, g\right) x(g)\right)$. Indeed, $U$ is an isometrey as 


$$
\begin{aligned}
\|\tilde{\Lambda}(\tilde{x})\|^{2} & =\widetilde{\varphi}\left(\tilde{x}^{*} \tilde{x}\right) \\
& =\int_{G} \varphi\left(x(g)^{*} x(g)\right) d g \\
& =\int_{G}\left\|\Lambda\left(\omega\left(g^{-1}, g\right) x(g)\right)\right\|^{2} d g \\
& =\|U(\tilde{\Lambda}(\tilde{x}))\|^{2} .
\end{aligned}
$$

Clearly, $U$ has dense range in $L^{2}(G ; \mathcal{H})$, so that $U$ extends to a unitary of $\widetilde{\mathcal{H}}$ onto $L^{2}(G ; \mathcal{H})$; we denote this extension by $U$ also.

Now for $\xi, \eta \in \widetilde{\mathscr{H}}$ and $\tilde{x}, \tilde{y} \in \tilde{\mathfrak{B}}$, we have

$$
\begin{aligned}
\langle\tilde{x} \tilde{y} \xi, \eta\rangle & =\int_{G} \int_{G}\langle\pi(g) I(x(g)) \pi(h) I(x(h)) \xi, \eta\rangle d h d g \\
& =\int_{G} \int_{G}\left\langle\pi(g) \pi(h) I\left(\alpha_{h}^{-1}(x(g)) x(h)\right) \xi, \eta\right\rangle d h d g \\
& =\int_{G} \int_{G}\left\langle\pi(g h) I\left(\alpha_{g h}^{-1}(\omega(g, h)) \alpha_{h}^{-1}(x(g)) x(h)\right) \xi, \eta\right\rangle d h d g \\
& =\int_{G} \int_{G}\left\langle\pi(k) I\left(\alpha_{k}^{-1}\left(\omega\left(g, g^{-1} k\right)\right) \alpha_{k^{-1}}(x(g)) y\left(g^{-1} k\right)\right) \xi, \eta\right\rangle d k d g \\
& =\int_{G} \int_{G}\left\langle\pi(k) I\left(\alpha_{k}^{-1}\left(\omega\left(k 1,1^{-1}\right)\right) \alpha_{1}(x(k 1)) y\left(1^{-1}\right)\right) \xi, \eta\right\rangle d 1 d k
\end{aligned}
$$

Thus $\tilde{x} \tilde{y}=\tilde{z}$, where $z(g)=\int_{G} \alpha_{g}^{-1}\left(\omega\left(g h, h^{-1}\right)\right) \alpha_{h}(x(g h)) y\left(h^{-1}\right) d h$. Hence we have

$$
\begin{aligned}
(U(\tilde{x} \tilde{\Lambda}(\tilde{y})))(g) & =(U(\tilde{\Lambda}(z)))(g) \\
& =\Lambda\left(\omega\left(g^{-1}, g\right) \int_{G} \alpha_{g}^{-1}\left(\omega\left(g h, h^{-1}\right)\right) \alpha_{h}(x(g h)) y\left(h^{-1}\right) d h\right) \\
& =\int_{G} \Lambda\left(\omega\left(g^{-1}, g\right) \alpha_{g}^{-1}\left(\omega\left(g h, h^{-1}\right)\right) \alpha_{h}(x(g h)) y\left(h^{-1}\right)\right) d h \\
& =\int_{G} \Lambda\left(\omega\left(h, h^{-1}\right) \omega\left(g^{-1}, g h\right) \alpha_{h}(x(g h)) y\left(h^{-1}\right)\right) d h .
\end{aligned}
$$

On the other hand, we may compute

$$
\begin{aligned}
& \left(\left(\int_{G} \lambda^{\omega}(h) I^{\alpha}(x(h)) d h\right) U(\tilde{\Lambda}(\tilde{x}))\right)(g) \\
= & \int_{G}\left(\lambda^{\omega}(h) I^{\alpha}(x(h)) U(\tilde{\Lambda}(\tilde{x}))\right)(g) d h \\
= & \int_{G} \omega\left(g^{-1}, h\right) \alpha_{g^{-1}}(x(h)) \Lambda\left(\omega\left(g^{-1} h, h^{-1} g\right) y\left(h^{-1} g\right)\right) d h \\
= & \int_{G} \Lambda\left(\omega\left(g^{-1}, h\right) \omega\left(g^{-1} h, h^{-1} g\right) \alpha_{g^{-1}}(x(h)) y\left(h^{-1} g\right)\right) d h \\
= & \int_{G} \Lambda\left(\omega\left(g^{-1}, g h\right) \omega\left(h, h^{-1}\right) \alpha_{h}(x(g h)) y\left(h^{-1}\right)\right) d h .
\end{aligned}
$$


Thus $U\left(\int_{G} \pi(g) I(x(g)) d g\right) U^{*}=\int_{G} \lambda^{\omega}(g) I^{\infty}(x(g)) d g$; an easy and well-known approximation argument now ensures that $U \pi(g) U^{*}=\lambda^{\omega}(g), g \in G$, and $U I(x) U^{*}$ $=I^{\alpha}(x), x \in \mathscr{M}$, and that $\{\mathscr{R}, I, \pi\}$ and $\left\{\mathscr{R}(\mathscr{M}, G, \alpha, \omega), I^{\alpha}, \lambda^{\omega}\right\}$ are isomorphic as extensions.

Q.E.D.

The strength of the concept of Plancherel pairs of weights lies in the requirement 5.2.1 (iii). This should be interpreted as an orthogonality condition (for the $\pi(g)$ ) over $I(\mathscr{M})$ ); it can also be interpreted as an analogue of a dimension condition frequently met with in the theory of (finite dimensional) central simple algebras.

From 5.2.2 we obtain immediately the following

Corollary 5.2.3. Let $\{\mathscr{R}, I, \pi\}$ be a normalized extension of $\{\mathscr{M}, \theta\}$ inducing $\alpha$ and $\omega$, and $\mathscr{M}$-G-equivalent with $\left\{\mathscr{R}(\mathscr{M}, G, \alpha, \omega), I^{\alpha}, \lambda^{\omega}\right\}$. Then the extensions $\{\mathscr{N}, I, \pi\}$ and $\left\{\mathscr{R}(\mathscr{M}, G, \alpha, \omega), I^{\alpha}, \lambda^{\omega}\right\}$ are isomorphic.

We also obtain the following more familiar criterion for a normalized extension to be a twisted crossed product (cf. [3],[12]).

Corollary 5.2.4. Let $\{\mathscr{l}, I, \pi\}$ be an extension of $\mathscr{M}$ by $G$, with $G$ discrete, and with $\{\Re, I, \pi\}$ inducing $\alpha, \omega$. Suppose further that each automonphism $\alpha_{g}, g \neq e$ is properly outer. Then if there is a normal, faithful, expectation E: $\Re \rightarrow I(\mathscr{M})$, the extensions $\{\mathscr{R}, I, \pi\}$ and $\left\{\mathscr{R}(\mathscr{M}, G, \alpha, \omega), I^{\omega}, \lambda^{\omega}\right\}$ are isomorphic.

Proof. We identify $\mathscr{H}$ with $I(\mathscr{M}) \subseteq \mathscr{N}$. Since for $g \neq e$ we have $E(\pi(g)) x$ $=E(\pi(g) x)=E\left(\alpha_{g}(x) \pi(g)\right)=\alpha_{g}(x) E(\pi(g))$, and $\alpha_{g}$ is properly outer, $E(\pi(g))=0$, $g \neq e$.

Thus if $\varphi$ is any faithful normal state on $\mathscr{M}$ and $\tilde{\varphi}=\varphi \circ E$, we may compute for $x \in \mathcal{K}(G ; \mathscr{M})$

$$
\begin{aligned}
\tilde{\varphi}\left(\tilde{x}^{*} \tilde{x}\right) & =\tilde{\varphi}\left(\sum_{g} \sum_{h} x(g)^{*} \pi(g)^{*} \pi(h) x(h)\right) \\
& =\sum_{g} \sum_{h} \varphi\left(x(g)^{*} E\left(\pi(g)^{*} \pi(h)\right) x(h)\right) \\
& =\sum_{g} \varphi\left(x(g)^{*} x(g)\right)
\end{aligned}
$$

thus $\{\varphi, \tilde{\varphi}\}$ Plancherel pair for $\{\mathscr{I}, \mathscr{M}, I, \pi\}$.

Q.E.D.

The relevant feature of the above proof of course is that $E(\pi(g))=0$ for $g \neq e$. 


\subsection{Dual Actions}

According to 3.1 .8 , if $G$ is an abelian group and $\{\Re, I, \pi\}$ any regular extension of $\mathscr{M}$ by $G$, the dual group $\hat{G}$ acts as automorphisms on $\mathscr{\Omega}$ in such a way that $\hat{\alpha}_{p}(\pi(g))=\overline{\langle g, p\rangle} \pi(g)$ and $I(\mathscr{M})=\left\{x \in \mathscr{R}: \hat{\alpha}_{p}(x)=x\right.$ for all $\left.p \in \hat{G}\right\}$. The following converse is patterned after the results of [18].

Proposition 5.3.1. Let $\Re$ be a von Neumann algebra, $G$ an abelian group, and $\pi: g \in G \rightarrow \pi(g) \in \mathcal{U}(\mathcal{I})$ a Borel map. Suppose there exists a continuous homomorphism $p \in \hat{G} \rightarrow \hat{\alpha}_{p} \in \operatorname{Aut}(\mathscr{R})$ with $\hat{\alpha}_{p}(\pi(g))=\overline{\langle g, p\rangle} \pi(g)$ for all $p \in \hat{G}$, $g \in G$, and set $\mathscr{M}=\left\{x: \hat{\alpha}_{p}(x)=x\right.$ for all $\left.p \in \hat{G}\right\}$. Then $\{\mathscr{M}, I, \pi\}$ is a regular extension of $\mathscr{M}$ of $G$, where $I$ is the inclusion map $I: \mathscr{M} \rightarrow \mathscr{M}$.

Proof. The fact that $\mathcal{M}$ and $\{\pi(g), g \in G\}$ generate $\mathscr{I}$ follows exactly as in [18]; we omit the details. Also, with $v(g, h)=\pi(g) \pi(h) \pi(g h)^{*}$ we see $\hat{\alpha}_{p}(v(g, h))$ $=\overline{\langle g, p\rangle} \overline{\langle h, p\rangle}\langle g h, p\rangle v(g, h)=v(g, h)$ so $v(g, h) \in \mathscr{M}$. Further, with $x \in \mathscr{M}$ we see $\hat{\alpha}_{p}\left(\pi(g) x \pi(g)^{*}\right)=\overline{\langle g, p\rangle} \pi(g) x \pi(g)^{*}\langle g, p\rangle=\pi(g) x \pi(g)^{*}$; thus if $\alpha_{g}(x)=\pi(g) x \pi(g)^{*}$ for $x \in \mathscr{M}$, then $\mathscr{M}$ is invariant under $\alpha_{g}$. We shall show that $\{\mathscr{l}, I, \pi\}$ is (isomorphic to) the regular extension constructed from $\alpha$ and $v$ as in 3.1.6.

Define a map $\delta: ~ \Re \rightarrow L^{\infty}(\hat{G} ; \Re)$ by $(\delta(y))(p)=\hat{\alpha}_{p}(y)$. Clearly $\delta$ is an isomorphism and $\delta(y)=y \otimes 1$ if and only if $y \in \mathscr{H}$; also $\delta(\pi(g))=\pi(g) \otimes \chi_{g}$ where $\chi_{g}$ denotes multiplication by the character $p \rightarrow \overline{\langle g, p\rangle}$ on $L^{2}(\hat{G})$.

We let $\mathscr{I}$ be represented faithfully on the Hilbert space $\mathscr{H}$; thus $L^{\infty}(\hat{G} ; \mathcal{H})$ is realized on $L^{2}(\hat{G} ; \mathcal{H})$. Let $\mathscr{F}$ be the unitary $\mathscr{F}: L^{2}(\hat{G} ; \mathcal{H}) \rightarrow L^{2}(G ; \mathcal{H})$ defined by

$$
(\mathscr{I} \xi)(g)=\int_{\hat{G}}\langle g, p\rangle \xi(p) d p ; \xi \in L^{2}(\hat{G} ; \mathcal{H})
$$

(We have chosen Haar measures on $G$ and $\hat{G}$ so that the Plancherel formula holds; $\mathscr{F}$ is nothing but the Fourier transform.) Setting $\delta(y)=\mathscr{F} \delta(y) \mathscr{F}^{*}$ for $y \in \mathscr{R}$ we obtain $\bar{\delta}(x)=x \otimes 1$ if and only if $x \in \mathscr{M}$ and $\delta(\pi(g))=\pi(g) \otimes \lambda(g)$, where $\lambda$ is the regular representation of $G$ on $L^{2}(G)$.

Define a unitary $U$ on $L^{2}(G ; \mathcal{H})$ by $(U \xi)(g)=\pi\left(g^{-1}\right) \xi(g)$. Now $(U(x \otimes 1)$ $\left.\times U^{*} \xi\right)(g)=\alpha_{g^{-1}}(x) \xi(g), x \in \mathscr{M}$ and

$$
\begin{aligned}
\left(U(\pi(g) \otimes \lambda(g)) U^{*} \xi\right)(h) & =\pi\left(h^{-1}\right) \pi(g)\left(U^{*} \xi\right)\left(g^{-1} h\right) \\
& =\pi\left(h^{-1}\right) \pi(g) \pi\left(h^{-1} g\right)^{*} \xi\left(g^{-1} h\right) \\
& =v\left(h^{-1}, g\right) \xi\left(g^{-1} h\right) .
\end{aligned}
$$


Thus the isomorphism Ad $U \circ \bar{\delta}$ carries $\mathscr{M}$ and $\pi(g), g \in G$ to the generators of the regular extension of $\mathscr{M}$ by $G$ determined by $\alpha$ and $v$. The proof is complete.

Q.E.D.

We note that if $G$ is discrete, 5.3.1 may be proven more easily using 5.2.4; the map $E: \mathscr{I} \rightarrow \mathscr{M}$ defined by $E(y)=\int_{\hat{G}} \hat{\alpha}_{p}(y) d p$ is a conditional expectation with $E(\pi(g))=0$ for $g \neq e$. The proof of 5.2.4 now applies.

\section{§6. Applications}

These fall in three (related) classes; we begin with twisted crossed products. Twisted crossed products have been seen to be useful since Connes [6] constructed examples of factors not anti-isomorphic with themselves (see [27] also for details), although they have been discussed in the literature much earlier ([32]); they have been advertized as a method of constructing von Neumann algebras which are not constructible using ordinary crossed products. However, we have

Theorem 6.1. Let $\mathscr{M}$ be properly infinite and $G$ arbitrary, or a $I I_{1}$-algebra and $G$ a finite group. Then any regular extension, and hence any twisted crossed product, of $\mathscr{M}$ by $G$, is already an ordinary crossed product.

Proof. Let $\{\mathscr{N}, I, \pi\}$ be a regular extension of $\mathscr{M}$ by $G$; by $4.3 .3\{\mathscr{R}, I, \pi\}$ is strongly equivalent to an extension $\left\{\Omega_{,} I, \pi_{1}\right\}$ where $\pi_{1}$ is a representation of $G$ in $\mathcal{U}(\mathcal{I})$. If we write Ad $\pi(g)(I(x))=I\left(\alpha_{g}(x)\right), \pi(g) \pi(h)=I(v(g, h)) \pi(g h)$, Ad $\pi_{1}(g)$ $\times(I(x))=I\left(\beta_{g}(x)\right)$ and $\pi_{1}(g)=I\left(u_{g}\right) \pi(g)$ for $g, h \in G$ and $x \in \mathcal{M}$, then $u_{g} \alpha_{g}\left(u_{h}\right)$ $v(g, h) u_{g h}^{*}=1$ and $\beta_{g}=\operatorname{Ad} u_{g} \circ \alpha_{g}, g, h \in G$.

We let $\mathscr{M}$ act on $\mathscr{H}$ and $\mathscr{N}$ on $L^{2}(G ; \mathscr{H})$; define a unitary $U$ on $L^{2}(G ; \mathcal{H})$ by $(U \xi)(g)=u_{g^{-1}} \xi(g)$. Then

$$
\begin{aligned}
\left(U I(x) U^{*} \xi\right)(g) & =u_{g^{-1} \alpha_{g^{-1}}(x) u_{g^{-1}}^{*} \xi(g)} \\
& =\beta_{g}^{-1}(x) \xi(g) \\
& =\left(I^{\beta}(x) \xi\right)(g), \quad x \in \mathscr{M}
\end{aligned}
$$

and

$$
\begin{aligned}
\left(U \pi(h) U^{*} \xi\right)(g) & =u_{g^{-1}} v\left(g^{-1}, h\right) u_{g-1}^{*} \xi\left(h^{-1} g\right) \\
& =u_{g^{-1}} \alpha_{g^{-1}}\left(u_{h}^{*}\right) u_{g-1}^{*} \xi\left(h^{-1} g\right) \\
& =\beta_{g}^{-1}\left(u_{h}^{*}\right) \xi\left(h^{-1} g\right) \\
& =\left(I^{\beta}\left(u_{h}^{*}\right) \lambda^{\iota}(h) \xi\right)(g), \quad h \in H,
\end{aligned}
$$


where ' denotes the trivial 2-cocycle. Thus $\mathscr{R}$ is isomorphic with $\mathscr{R}(\mathscr{M}, G, \beta)$.

Q.E.D.

The interpretation of this in terms of non-anti-isomorphism is that there are von Neumann algebras $\mathscr{M}$ anti-isomorphic with themselves, and representations $g \in G \rightarrow \alpha_{g} \in \operatorname{Aut}(\mathscr{M})$ such that for no anti-automorphism $\sigma$ of $\mathscr{M}$ is $\sigma \circ \alpha_{g} \circ \sigma^{-1}=\alpha_{g}$ for all $g \in G$. Interest in twisted crossed products is thus reduced to abelian algebras and possibly $\mathrm{II}_{1}$ (non-factor) von Neumann algebras.

We now turn to extensions of injective von Neumann algebras.

Theorem 6.2. Let $\{\mathscr{l}, I, \pi\}$ be an extension of an injective von Neumann algebra $\mathscr{M}$ by $G$. Then $\mathscr{N}$ is injective if either $\mathscr{M}$ is a $I_{1}$-algebra and $G$ is finite, or if $\mathscr{M}$ is properly infinite, and $G$ contains a dense, countable, amenable subgroup.

Proof. Follows from 4.3.3 and [7].

Q.E.D.

In particular, any extension of the hyperfinite factor by a finite group is injective.

If $M$ is any locally compact group, and $\rho$ a continuous unitary representation of $M$ on separable Hilbert space, we say $\rho$ is A.F.D. (approximately finite dimensional) if $\rho(M)^{\prime \prime}$ is an injective von Neumann algebra. If all representations of $M$ are A.F.D, we say $M$ itself is A.F.D.

Theorem 6.3. Let $1 \rightarrow M \rightarrow N \rightarrow G \rightarrow 1$ be an exact sequence of locally compact separable groups, where $G$ contains a dense, countable, amenable subgroup; let $\rho$ be a representation of $N$. Then

(i) if $\rho(M)^{\prime \prime}$ is properly infinite and injective, $\rho$ is A.F.D.,

(ii) if $\rho(M)^{\prime \prime}$ is the hyperfinite factor and $G$ finite, $\rho$ is A.F.D.,

(iii) if $M$ is connected, $N$ is A.F.D.

Proof. i) and ii) are trivial. Since any representation of a connected group generates a properly infinite ([17]), injective ([7]) von Neumann algebra, (iii) follows.

We note it is not clear whether or not, if $\rho$ is a representation of $M$ which is A.F.D, the induced representation $\operatorname{Ind}_{M}^{N} \rho$ of $M$ is also injective.

\section{References}

[1] Auslander, L. and Moore, C. C., Unitary representations of solvable Lie groups, Memoirs of A.M.S., 62, 1966. 
[2] Christensen, J. P. R., Topology and borel structure, North Holland mathematics studies, 10, 1974.

[3] Connes, A., Une classification des facteurs de type III, Annales de l'Ecole Normale Superieure, 6 (1973), 18-252.

[4] - Almost periodic states and factors of type $\mathrm{III}_{1}, J$. Functional Analysis, 16 (1974), 415-445.

[5] — Periodic automorphisms of the hyperfinite factor of type II, Queen's preprint, 1974-25.

[6] —, A factor not anti-isomorphic with itself, Ann. Math., 101 (1975), 536-554.

[7] ——, Classification of injective factors, Ann. Math., 104 (1976), 73-116.

[8] - Outer conjugacy of automorphisms of factors, Proc. Meeting on $C^{*}$ algebras and their application to theoretical physics, 1975, Rome.

[9] Connes, A. and Takesaki, M., The flow of weights on a factor of type III, Tôhoku Math. J., 29 (1977), 437-575.

[10] Dixmier, J., Les $C^{*}$-algebres et leurs representations, Gauthier-Villars, Paris, 1964.

[11] Effros, E., The Borel space of von Neumann algebras on a separable Hilbert space, Pac. J. Math., 15 (1965), 1153-1164.

[12] Feldman, J. and Moore, C. C., Ergodic equivalence relations von Neumann algebras, and cohomology, I, II, Trans. Amer. Math. Soc., 234 (1977), 289-324 and 325-359.

[13] Fell, J. M. G., An extension of Mackey's method to Banach *-algebraic bundles, Memoirs of A.M.S., 90, 1969.

[14] Haagerup, U., The standard form of von Neumann algebras, Math. Scand., 37 (1975), 271-283.

[15] Hahn, P., Haar measure for measure groupoids, Trans. Amer. Math. Soc., 242 (1978) 35-73.

[16] Kadison, R., Transformations of states in operator theory and dynamics, Topology, 3 (1965), 117-198.

[17] Kadison, R. and Singer, I. M., Some remarks on representations of connected groups, Proc. Nat. Acad. Sci., U.S.A., 38 (1952), 419-423.

[18] Landstad, M., Duality theory of covariant systems, Trans. Amer. Math. Soc., 248 (1979), 223-267.

[19] Mackey, G. W., Point realization of transformation groups, Illinois J. Math. 6 (1962), 327-335.

[20] Mackey, G. W., Unitary representations of group extensions, Acta Math., 99 (1958), 265-311.

[21] MacLane, S., Homology, Academic Press, 1963.

[22] Moore, C. C., Group extensions and cohomology for locally compact groups III, Trans. Amer. Math. Soc., 221 (1976) 1-36.

[23] Nakamura, M. and Takeda, Z., On the extensions of finite factors, Proc. Japan Academy 35 (1959), 149-154.

[24] Nielsen, O. A., Borel sets of von Neumann algebras, Amer. J. Math., 95 (1973), 598607.

[25] Paschke, W., Inner product modules arising from compact automorphism groups of von Neumann algebras Trans. Amer. Math. Soc., 224 (1976), 87-102.

[26] Sutherland, C. E., Crossed products, direct integrals and Connes classification of type III factors, Math. Scand. 40 (1977), 209-214.

[27] Cohomology and extensions of von Neumann algebras I, Publ, RIMS, Kyoto Univ., 16 (1980), 105-133.

[28] Suzuki, N., Crossed products of rings of operators, Tohoku Math. J., 1 1 (1959), 113124. 
[29] Takesaki, M., Tomita's theory of modular Hilbert algebras and its applications, Lecture Notes in Math., 128, Springer, 1970.

[30] — Duality for crossed products and the structure of von Neumann algebras of type III, Acta Math. 131 (1974), 249-310.

[31] - Relative commutant theorem in crossed products and the exact sequence for the automorphism group of a factor of type III, Proc. Meeting on $C^{*}$-algebras and their application to theoretical physics, 1975, Rome.

[32] Zeller-Meier, G., Produits croisés d'une $\mathrm{C}^{*}$-algebra by a group of automorphisms, J. Math. Pure et Appl., 47 (1968), 101-239. 